\title{
Overcoming interference from hydrolysable cations during the determination of sulphuric acid by titration
}

by

\section{Pravani Pillay}

Submitted in partial fulfilment of the requirements for the degree

\section{- MAGISTER SCIENTIAE}

In the Faculty of Natural, Agricultural and Information Sciences

University of Pretoria

Pretoria

November 2000 
Die oorkoming van steurings veroorsaak deur hidroliseerbare katione in die bepaling van swaelsuur met titrasie

deur

Pravani Pillay

Studieleier: Professor Jacobus F. van Staden

Department Chemie

Universiteit van Pretoria

Graad: Magister Scientiae 


\section{Synopsis}

During the neutralisation of mineral acids, hydrolysable cations precipitate and consume the titrant. Depending on the concentration and type of cation, this consumption leads to erroneously high titration results. Various methods have been suggested to overcome this interference, but all suffer from certain limitations - especially at high cation to free acid ratios. A simple and practical method is suggested to overcome the effect at high cation concentrations. It is based on the addition of neutral salt to enhance the activity of the protons, which coincides with a shift in the inflection of the titration curve. This results in a shift of the titration end point to a lower $\mathrm{pH}$, and a better separation of the free acid and cation curve. This effect, combined with performing the titration in a methanolic environment, decreases the hydrolysation of the cations and improves the inflection of the titration curve to such an extent that more than a ten-fold excess of iron(III) concentration (the most serious interference) could safely be tolerated during the free acid titration. 


\section{Samevatting}

Gedurende die neutralisasie van mineraalsure, presipiteer die hidroliseerbare katione met verbruik van die titrasie reagens. Afhangende van die konsentrasie en aard van die katioon, gee dit aanleiding tot verkeerde resultate. Verskillende metodes is voorgestel om die steuring te oorkom. Elkeen het egter sy eie beperkings - veral in oplossings waar 'n hoë katioon tot vry suur verhoudings voorkom. 'n Eenvoudig en praktiese metode word voorgestel om die effek te oorkom. Dit is gebaseer op die byvoeging van neutrale soute om die aktiwiteit van die protone te verhoog, wat ' $n$ verskuiwing van die infleksie punt van die titrasie-kurwe tot gevolg het. Die titrasie eindpunt verskuif ook na 'n laer $\mathrm{pH}$, en veroorsaak ' $n$ beter skeiding van die vry suur en katioon kurwes. Die infleksiepunt van beide titrasie kurwes kan verder verbeter word deur die titrasies in metanol te doen. Die eindresultaat is, suur kan bepaal word in oplossings waar die yster(III) tot suur verhouding tien maal hoër is as wat voorheen die geval was. 


\section{Acknowledgements}

I would like to thank the following people who have contributed to the success of this project:

To Dr Deon Barnes, I would like to express my sincere gratitude to you for your kindness, patience, and guidance. You listened and encouraged me, without telling me what to do. Thank You.

To Dr Elaine Jones-Watson, the opportunities you afforded me gave me the confidence to take on this project. Thank you for your guidance, and encouragement.

To Professor Jacobus van Staden, thank you for allowing me to do this project with you as supervisor.

To my parents, you have enabled me to have an education, which you yourselves never had. Thanks to you I have achieved this academic milestone. I will be eternally grateful.

To Ravi, I appreciate your love, support and understanding during my studies.

Thanks to MINTEK for the financial support and the time allowed for completion of this project. 


\section{TABLE OF CONTENTS}

Synopsis

Samevatting

ii

Acknowledgements

Table of Contents

\section{Chapter 1: Introduction}

1.1 Interferences 3

1.2 Origin of Samples 4

1.3 Sulphuric Acid-Properties, Importance

1.4 Overcoming Interference $\quad 9$

\section{Chapter 2: Theory}

2.1 The Nature of Acids and Bases $\quad 15$

$2.2 \mathrm{pH} \quad 16$

2.3 Theory of Acid-Base Titrations 17

2.4 Titrations 18 
2.5 Neutralisation Indicators

2.6 Titration/Neutralisation Curves

2.7 Strong/Weak Acids and Bases

2.8 Titration Curves for Polyprotic Acids

2.9 Acid-Base Concepts

2.10 Hard and Soft Acids and Bases

2.11 Acid-Base Strength and Hardness and Softness

2.12 Potentiometry

2.13 Relation between Potential and $\mathrm{pH}$

2.14 Automatic Potentiometric Titrations

2.15 Endpoint Location

2.16 Automatic Titrators

\section{Chapter 3: Experimemtal}

3.1 Apparatus

3.2 Reagents

42

\section{Chapter 4: Results and Discussion}

4.1 Establishing Interferences

4.1.1 Effect of Copper(II), Nickel(II) and Cobalt(II) on Acid

Determinations

4.1.2 Effect of Iron(II) on Acid Determination 
4.1.3 Effect of Manganese(II) on Acid Determination

4.1.4 Effect of Iron(III) on Acid Determination

4.1.5 Conclusion

4.2 Effect of Complexing Agents

4.2.1 Potassium Oxalate as Complexing Agent

4.2.2 Potassium Tartrate as Complexing Agent

4.2.3 Failure of Complexometric Methods

4.2.4 Conclusion

4.3 The Effect of Dilution on Acid Determination

4.4.1 Lithium Chloride Titrations

4.4.2 Evaluation of Other Neutral Salts

4.4.3 Conclusion

4.5.1 Cyclohexylamine

4.5.2 Methanol

81

4.5.3 Pyridine

4.5.4 Acetone 
4.6 Neutral Salt in a Non-aqueous Medium

4.6.1 Conclusion

92

4.7 Analysis of Samples, Detection Limits

4.7.1 Conclusion

94

4.8 Figures of Merit

96

4.8.1 Accuracy

96

4.8.2 Precision

98

4.8.3 Conclusion

100

Chapter 5: Conclusion

101

References

105 


\section{Chapter 1: Introduction}

Many hydrometallurgical processes make use of sulphuric acid [1]. Electroplating processes utilise the $\mathrm{pH}$ buffering capabilities of the acid, while leaching processes use the fact that the oxidation potential of a process can be controlled with sulphuric acid. In these processes, the concentration of the acid needs to be controlled within tight pre-set concentration ranges, and the precise and reproducible determination of the concentrations of the acid is of utmost importance. Being metallurgical processes, the concentration of cations is high and this causes interference in determining the acid concentration.

During the acid-base titration, the hydrolysable cations form basic salts or hydroxides, which consume part of the titrant [2,3]. With conventional methods the loss of titrant due to this hydrolysation is indistinguishable from the titrant necessary for the neutralisation of the sulphuric acid, and the titrations are erroneously high. The problem is magnified as the ratio of hydrolysable cation to free acid increases, particularly to ratios higher than one.

Although various methods have been reported to determine the concentration of sulphuric acid in the presence of hydrolysable cations, no universal methods exist for this determination [4-18]. The methods are usually specific to a defined matrix and can be classified into five main groups according to the strategy employed:

- addition of a complexometric reagent [4-10] 
- removal or reducing of concentration, of the interfering cation in the aqueous phase to be analysed [4]

- electronic or mathematical resolution of the inflections on the titration curve [7]

- use of mathematical derivative techniques [7]

- Gran Plots $[9,10,17,18]$

The reported methods are able to tolerate an iron(III) to free acid ratio of $2: 1$. The samples usually analysed at MINTEK have a higher iron(III) to free acid ratio. To compound the problem, the samples also contain high concentrations of copper(II), iron(II), manganese(II), cobalt(II), and nickel(II).

It has been reported [13-16] that changing the medium in which one performs the titration can alter the activity of the proton and cations. Lithium chloride has been suggested as a neutral salt that would enhance the activity of the proton thereby reducing the interference from the hydrolysable cations.

The aim of this investigation was to find a method that allows for the titration of sulphuric acid in the presence of hydrolysable cations such that the titration results are indicative of acid content only. The investigation explored the use and the possible application of lithium chloride as a neutral salt to enhance the determination of free acid in the presence of hydrolysable cations (in hydrometallurgical samples). This project included an investigation into other neutral salts as well as various organic media as a means of improving the acid titrations in the presence of hydrolysable cations. 


\subsection{Interferences}

The problems experienced during the titration of free acid in the presence of hydrolysable cations are clearly influenced by the hydration of certain cations [2,3,19]. Most soluble metal salts do not have discrete metal ligand bonds when they are in solution as the cation is enclosed in a hydration shell. When hydrolysable cations are placed in a basic environment, the cation undergoes hydrolysis and a basic salt or hydroxide form. A simplified model for the hydrolysis reaction is given by Equation 1.1, where $\mathrm{M}$ is the respective cation, $\mathrm{n}$ is the co-ordination number, and $\mathrm{z}$ is the charge of the cation.

$$
\mathrm{M}\left(\mathrm{H}_{2} \mathrm{O}\right)_{\mathrm{n}}{ }^{{ }^{+}}+\mathrm{H}_{2} \mathrm{O} \quad \rightleftharpoons \quad\left\{\mathrm{M}\left(\mathrm{H}_{2} \mathrm{O}\right)_{\mathrm{n}-1}(\mathrm{OH})\right\}^{(\mathrm{z}-1)+}+\mathrm{H}_{3} \mathrm{O}^{+}{ }_{(\mathrm{aq})}
$$

When the cation is hydrated, it will act as a source of protons. The chemical equilibrium changes as the $\mathrm{pH}$ changes and, as the solution becomes more basic, more protons are released. This additional source of protons contributes to an erroneous titration result for free acid. In order to find a method that addresses this interference knowledge of the sample matrix, and the previous research carried out in this area was necessary. 


\subsection{Origin of Samples}

For an understanding of the sample matrix the origin of the sample must be known. Many hydrometallurgical processes make use of sulphuric acid in some form or another $[1,20,21]$. Electroplating processes utilise the $\mathrm{pH}$ buffering capabilities of the acid, and leaching processes make use of the fact that the oxidation potential can be controlled with the use of sulphuric acid. In these processes the concentration of the acid needs to be controlled within tight precept concentration ranges and the precise and reproducible determination of the concentration of the acid in the presence of hydrolysable cations is of utmost importance.

The two main steps of hydrometallurgical flow sheets are leaching, or dissolution of the metal in a suitable aqueous solvent, and recovery or precipitation of the metal or its compounds from solution (Figure 1.1) [20,21]. There are usually several more steps involved in the leaching/dissolution and purification before the recovery of pure metal becomes feasible. The solvents used in these processes are usually regenerated and recycled. 
Figure 1.1: Generalised flow of hydrometallurgical processes.

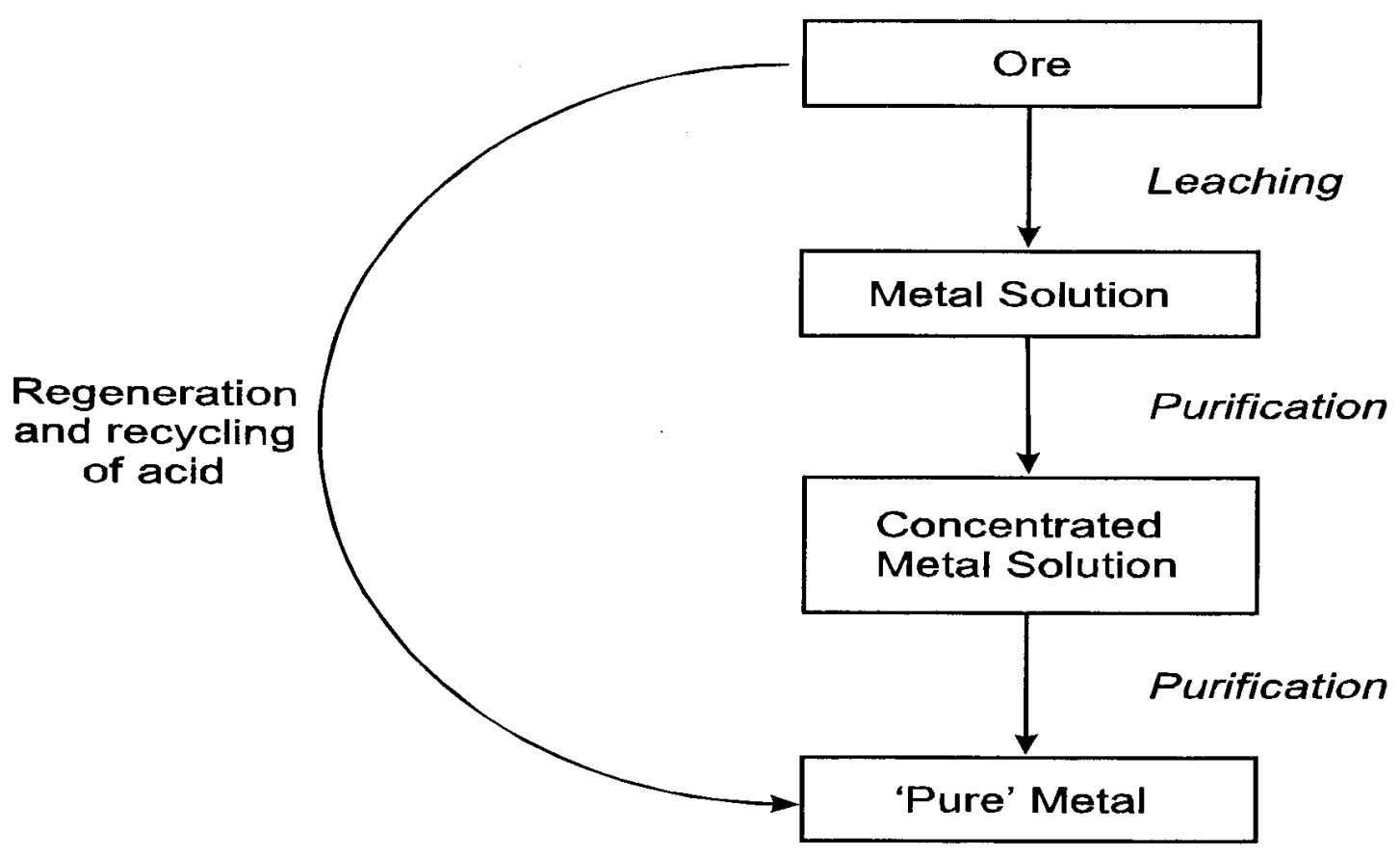

\section{Leaching}

The purpose of the leaching operation is to dissolve the desired mineral and to separate the desired metal from the gangue material. The reaction should be selective and fast, and the solvent inexpensive and easily regenerated. Several leaching agents are commonly used. The most important solvent for oxide ore and for dead-roasted sulphide concentrates is dilute sulphuric acid. Other acids can also be used, but are seldom employed because of higher costs and corrosion problems.

\section{Recovery of Metal from Solution}

The aqueous metal bearing leach solution is further refined by one of three processes. 
Electrowinning: This is the recovery of a metal by electrochemical reduction of one of its compounds dissolved in as suitable electrolyte. Various types of solutions can be used, but sulphuric acid is preferred because it is less corrosive than other inorganic acids and it is inexpensive. Also the high mobility of the hydrogen ion leads to high conductivity. Along with metal deposition at the cathode, acid is produced at the anode which can be recycled into the leaching step.

Precipitation: The desired metal could also be separated from impurities selected by precipitation. Metal hydroxides precipitate at different $\mathrm{pH}$ values. Hence by increasing or decreasing the $\mathrm{pH}$ of the solution different metal hydroxides can be precipitated.

Solvent Extraction: The desired metal can also be purified by solvent extraction. The desired metal is extracted onto a column and then eluted into a solution from which it is recovered by electrowinning. Once again a sulphuric acid solution is the preferred solvent.

Hydrometallurgical processing solutions have widely different matrices. There are however similarities. The most common solutions requiring acid analyses by the MINTEK Analytical Science Division contained manganese(II), iron(II), iron(III), cobalt(II), nickel(II) and copper(II). The solutions mostly contained sulphuric acid with high cation content. 


\subsection{Sulphuric Acid - Properties, Importance}

Sulphuric acid has been known as early as the eighth century where the Arabians referred to it as "dissolving water" formed by the distillation of nitre with vitriol [23]. The acid is sometimes called oil of vitriol or vitriolic acid because it is was initially derived from a metal sulphate. Metal sulphates were referred to as vitriols [23].

Sulphuric acid is a strong mineral acid with the formula $\mathrm{H}_{2} \mathrm{SO}_{4}$. It is colourless, transparent, very viscous liquid. $98 \%$ pure sulphuric acid has a melting point of $3^{\circ} \mathrm{C}$, a boiling point of $338^{\circ} \mathrm{C}$ with a density of 1.834 at $25^{\circ} \mathrm{C}$ [20-22].

Sulphuric acid reacts vigorously with water to form several hydrates. The concentrated acid therefore acts as an efficient drying agent, taking up moisture from the air. It can also extract water from compounds such as sugar and starch. Because of the hydrate formation a great amount of heat is evolved when sulphuric acid is mixed with water.

The concentrated acid acts as a strong oxidising agent due to its tendency to loose an atom of oxygen to form sulphurous acid.

Sulphuric acid is both a strong acid and a weak base with characteristic hygroscopic and oxidising properties. The sulphate ion, is chemically and thermally very stable. Dilute sulphuric acid is the preferred electrolyte for industrial metal electrowinning and 
electroplating plants on account of its high conductivity and the chemical stability of the sulphate ion.

Dilute sulphuric acid, being a strong diprotic acid, will dissolve most base metals, however barium and lead are exceptions. Hydrogen is released and the respective metal sulphates and bisulphates are formed. Hot concentrated sulphuric acid has an oxidising effect, reacting with precious metals and with carbon, phosphorus, and sulphur, which is reduced to sulphur dioxide.

The world's production of sulphuric acid stands at 5 million metric tons and a large amount of it is used in the manufacture of fertilisers [23]. The concentrated acid also decomposes salts of most other acids. It is therefore widely used in the preparation of other acids. Various sulphates of metals, starch and sugar are manufactured with the aid of sulphuric acid. The concentrated acid is also used in the manufacture of explosives. 


\subsection{Overcoming Interference}

To develop a method to overcome the interference by hydrolysable cations an understanding of existing methods is required. Of the seven procedures on file at Mintek based on previous research[4-16], two are based on the formation of complexes with the hydrolysable cations (the potassium oxalate method and the fluoride method). The lithium chloride method is based on the enhancement of the activity of hydrogen ions. The acetone and acetone-potassium iodide methods are based on the removal of salts by the reduction of their solubility in acetone. The potassium iodide method is based on the reduction of iron(III) to a less easily hydrolysable cation.

The potassium oxalate method depends on the ability of the oxalate ion to form stable complexes with many cations. When the free acid is titrated with sodium hydroxide solution, the stability of these complexes prevents hydrolysis at the $\mathrm{pH}$ value of the neutral point.

In the lithium chloride method the addition of concentrated solutions of salts of strong acids and strong bases to a solution appears to enhance the activity of the hydrogen ions. This enhancement manifests itself as a decrease in the $\mathrm{pH}$ value of dilute acid solutions. The hydrolysis of these cations forming chloro-complexes, for e.g. iron(III), copper(II), and zinc(II) is also minimised. 
In the potassium iodate method the hydrogen ions in the presence of iodate and iodide ions cause the liberation of elemental iodine according to:

$$
\mathrm{IO}_{3}^{-}+5 \mathrm{I}^{-}+6 \mathrm{H}^{+} \rightarrow 3 \mathrm{I}_{2}+3 \mathrm{H}_{2} \mathrm{O}
$$

The amount of iodine that is liberated is proportional to the concentration of hydrogen ions that were present originally, and the concentration of these ions can therefore be calculated from a determination of the liberated iodine.

The acetone method depends on the reduction of the solubility of the salts of hydrolysable cations by the addition of acetone, and the subsequent removal of the precipitated salts by filtration. The free acid is then determined by titration.

The acetone- potassium iodide method is a modification of the acetone method. Since the acetone method failed in the presence of iron(III) it was thought that the reduction of iron(III) by potassium iodide would reduce or eliminate the adverse effects of the appreciable soluble iron(III) sulphate in the aqueous-acetone mixture.

In the potassium iodide method, iron(III) is reduced to iron(II) by potassium iodide. The liberated iodine is reacted with an excess of sodium thiosulphate. The free acid is than titrated with minimal interference from ferrous hydroxide which precipitates at $\mathrm{pH}$ of about 6. 
In the presence of a small excess of fluoride ions, iron(III) is not precipitated until all the free acid has been neutralised by the titrant. When the free acid has been neutralised, hydrated iron(III) oxide begins to precipitate, indicating that the neutral point has been reached. Separan NP10, a commercial coagulant, is used to coagulate the precipitate, and this makes the neutral point clearer to observe.

From the experiments carried out by Solomons [4] it was concluded that none of the methods were satisfactory for the determination of free acid in the presence of high concentrations of cation and low concentration of acid. The potassium oxalate and lithium chloride methods however yielded usable endpoints with satisfactory results for the solutions examined. Crithchfield and Johnson $[13,14]$ however reported that lithium chloride could be used for acid analysis in the presence of hydrolysable cations. Various authors $[6,11,12]$ reported the success of the potassium oxalate method in different sample solutions.

Another method for determining an endpoint of an acid-base titration is that published by Baumann et al [6]. Their method is based on a Gran Plot $[10,17,18]$, and depends on the fact that the addition of free acid reverses the equilibrium represented in equation 1.1 . Standard additions of acid are made to the analyte solution, such that the hydrolysis is reversed. The hydrolysis is suppressed further by the addition of potassium thiocyanate. If the solution contains no hydrolysable species, then the addition of acid causes the plot of $\mathrm{pH}$ vs. the volume of titrant to be linear. In the presence of interferences, the curve does not have a Nernstian slope until hydrolysis has been completely reversed. During 
the time represented by the non-linear section of the curve, the added protons are consumed in reversing the hydrolysis reaction and do not contribute solely towards the changing of $\mathrm{pH}$. A parameter, $\phi$, can be defined in terms of the initial volume of the sample, the volume of titrant added, and the $\mathrm{pH}$ value. Before the equivalence point, the parameter $\phi$ is defined as:

$$
\phi=(\text { initial volume }+ \text { added volume }) \times 10^{(-\mathrm{pH})}
$$

after the equivalence point, a related $\phi^{\prime}$ is defined as:

$$
\phi^{\prime}=(\text { initial volume }+ \text { added volume }) \times 10^{(\mathrm{pH})}
$$

A modification of the original Gran Plot method was used by Baumann et al [6]. for the determination of free acid in the presence of hydrolysable cations. In this modification, the acid solution was 'titrated' with standard acid, and consequently equation 1.3 is applicable. The parameter $\phi$ when plotted vs. the titrant volume is found to be linear, or to approach linearity, when acid is added. The linearity of equation 1.3 is a first approximation, which is valid if the increments in the volume of titrant are small compared to those in the total volume of the system. The assumptions and derivations are detailed in Gran's work [17,18]. Expected results of the method for the determination of free acid in the presence of hydrolysable cations are given in Figure 1.2. Figure 1.3 shows all possible occurrences for the system with the addition of standard acid, based on the principles that Gran formulated. The intersection of the extrapolation of the linear section 
and the $\mathrm{x}$-axis $(\phi=0.0)$ represents the equivalence point for the amount of free acid. A negative intercept indicates the equivalence volume of free acid initially present in the system.

Its slowness and number of calculations hamper Gran's method. Baumann et al [6] showed that molar concentrations of metal ion up to 2.5 times that of the acid concentration could be tolerated. A level which is still too low for samples analysed in this laboratory.

Figure 1.2: Gran plot for free acid concentrations in the presence of hydrolysable cations

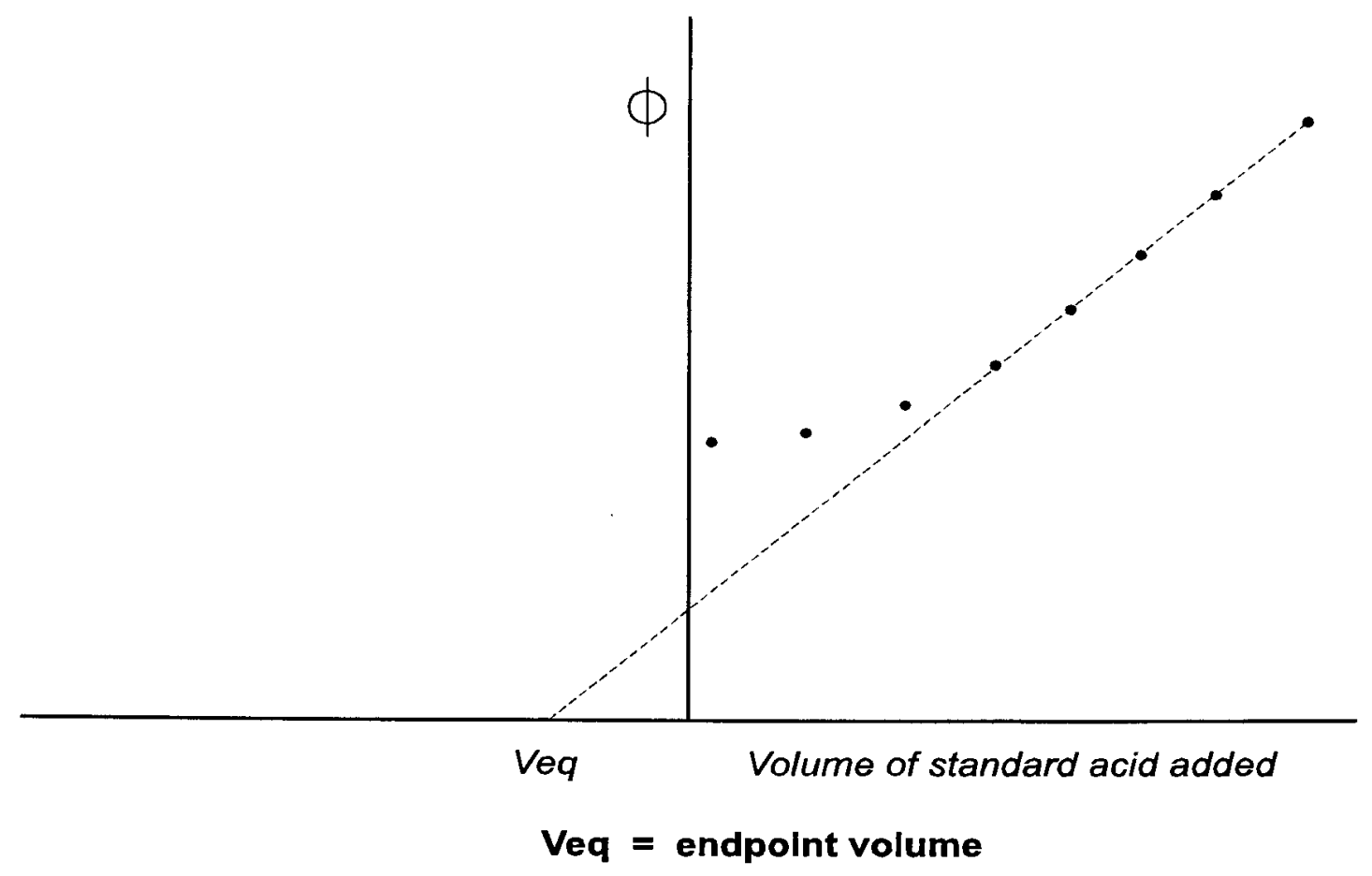


Figure 1.3: Gran Plots for all possible occurrences of acidic, neutral, or basic solutions in the presence or absence of hydrolysable cations, indicating the endpoint volume, $\mathrm{V}_{\mathrm{eq}}$, of acid or base.

\section{No Hydrolysable cations present}

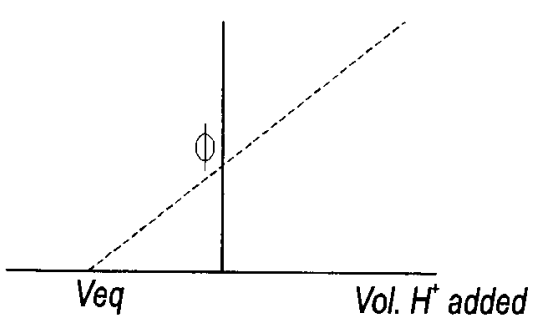

Excess Acid

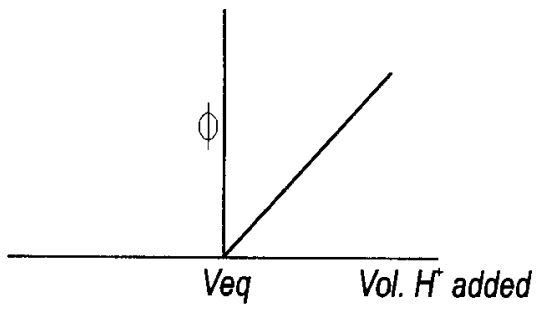

Neutral Solution

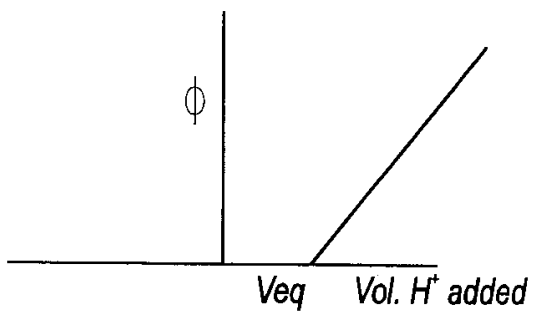

Excess Base

\section{Hydrolysable} cations present
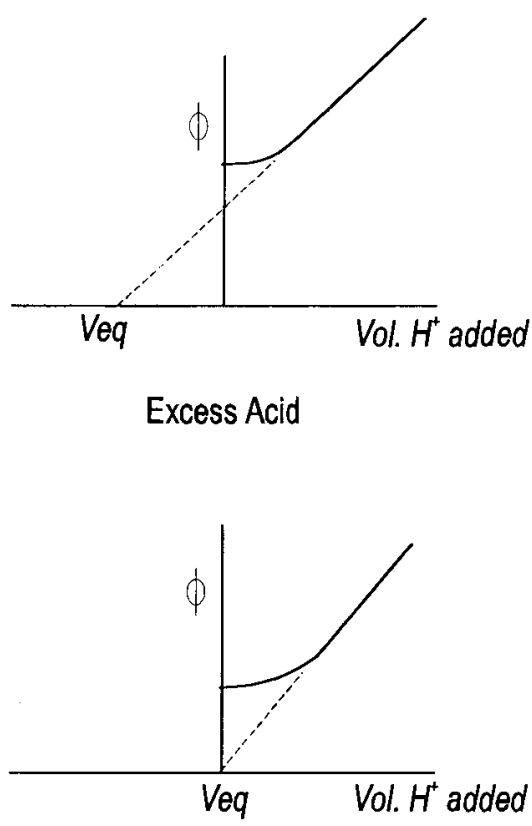

Neutral Solution

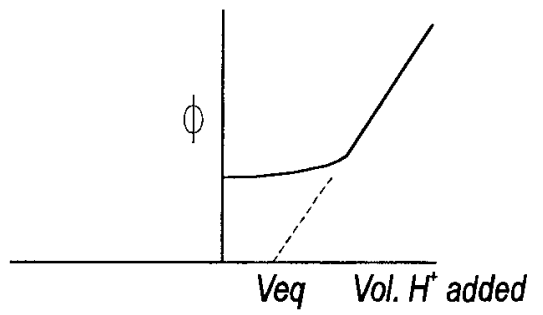

Excess Base 


\section{Chapter 2: Theory}

\subsection{The Nature of Acids and Bases}

A better understanding of acids and bases are gained by looking at water and the theory arising from this to give various definitions used in acid - base theory [24-33].

Water dissociates into ions according to the following equation:

$\mathrm{H}_{2} \mathrm{O} \quad \rightleftharpoons \quad \mathrm{H}^{+}(\mathrm{aq})+\quad \mathrm{OH}^{-}(\mathrm{aq})$

The equilibrium of this reaction lies far to the left. Hence at any given moment only 1 in 10000000 water molecules are dissociated. According to the laws of equilibrium the equilibrium expression for the above reaction can be formulated as:

$$
\mathrm{K}_{\mathbf{c}}=\left[\mathrm{H}^{+}\right]\left[\mathrm{OH}^{-}\right] /\left[\mathrm{H}_{2} \mathrm{O}\right]
$$

In dilute aqueous solutions the concentration of water is large and remains essentially constant. Its concentration can therefore be included in $\mathrm{K}_{\mathrm{c}}$ for dilute solutions. The equilibrium expression then simplifies to:

$$
\mathrm{K}_{\mathrm{w}}=\left[\mathrm{H}^{+}\right]\left[\mathrm{OH}^{-}\right]=1.0 \times 10^{-14}
$$


The dissociation of water is given the symbol $\mathrm{K}_{\mathrm{w}}$ and is very small.

According to the equation any aqueous solution at $25^{\circ} \mathrm{C}$ will contain $\mathrm{H}^{+}$and $\mathrm{OH}^{-}$ions at such concentrations that their product will be $1.0 \times 10^{-14}$. In pure water the hydroxide and hydrogen ions will be present in equal amounts.

Thus in pure water: $\left[\mathrm{H}^{+}\right]=\left[\mathrm{OH}^{-}\right]=1.0 \times 10^{-7} \mathrm{~mol} / \mathrm{l}$

A solution in which $\left[\mathrm{H}^{+}\right]$equals $\left[\mathrm{OH}^{-}\right]$is said to be neutral. A solution in which the hydrogen ion concentration is larger than the hydroxide ion concentration is said to be acidic. A basic solution has a hydroxide ion concentration that is higher than the hydrogen ion concentration. As the concentration of the one ion increases the concentration of the other decreases proportionally so that equation 2.3 is satisfied. This implies that a solution where the hydrogen ion concentration is greater than $1.0 \times 10^{-7}$ $\mathrm{mol} / \mathrm{l}$ is acidic; a solution where the hydroxide ion concentration is greater than $1.0 \times 10^{-7}$ $\mathrm{mol} / 1$ is basic.

\section{$2.2 \mathrm{pH}$}

The above information allows a quantitative description of acidity and basicity of a solution to be made by specifying the hydrogen ion concentration [24-33]. Sörensen proposed an alternative method of accomplishing this purpose, making use of a term known as $\mathrm{pH}$, defined as follows: 


$$
\mathrm{pH} \equiv-\log _{10}\left[\mathrm{H}^{+}\right]=\log _{10} 1 /\left[\mathrm{H}^{+}\right]
$$

Many solutions have a $\mathrm{pH}$ between 1 and 14, corresponding to a variation in hydrogen ion concentration of $10^{-1}$ to $10^{-14} \mathrm{~mol} / \mathrm{l}$. For such solutions it is sometimes convenient to express acidity in terms of $\mathrm{pH}$ rather than hydrogen ion concentration, thereby avoiding the use of either small fractions or negative exponents. It follows from the definition that the lower the $\mathrm{pH}$, the more acidic a solution is; conversely, the higher the $\mathrm{pH}$, the more basic is the solution. As the distinction is made between neutral, acid, and basic solutions on the basis of the hydrogen or hydroxide ion concentration, the same distinction can be made using $\mathrm{pH}$ :

$$
\begin{array}{ll}
\text { neutral solution: } & {\left[\mathrm{H}^{+}\right]=10^{-7} \mathrm{~mol} / \mathrm{l}, \mathrm{pH}=7.0} \\
\text { acidic solution: } & {\left[\mathrm{H}^{+}\right]>10^{-7} \mathrm{~mol} / \mathrm{l}, \mathrm{pH}<7.0} \\
\text { basic solution: } & {\left[\mathrm{H}^{+}\right]<10^{-7} \mathrm{~mol} / \mathrm{l}, \mathrm{pH}>7.0}
\end{array}
$$

\subsection{Theory of Acid-Base Titrations}

Neutralisation reactions include the titration of free bases, or those formed from salts of weak acids by hydrolysis with a standard (acidimetry), and the titration of free acids, or those formed by the hydrolysis of salts of weak bases, with a standard base (alkalimetry) [24]. These reactions involve the combination of hydrogen and hydroxide ions to form water. 


\subsection{Titrations}

The basis of a titration is the determination of a solution of unknown concentration with a solution of known concentration (called a standard) [24-33]. The standard solution is added to the unknown solution until the reaction is just complete. This process is termed a titration. The point at which the reaction is complete is called the equivalence point (theoretical or stoichiometric end-point).

The completion of the titration should be detectable by some change. This change is usually visual and is the change produced by an auxillary reagent i.e. an indicator. The change is a colour change or turbidity. The point at which this occurs is called the endpoint.

To carry out a titration the following conditions must be met:

1. The reaction must be simple and expressed by a chemical equation, the determined substance must react completely with the reagent stoichiometrically

2. The reaction must be fast

3. There must be a distinguishable change in free energy leading to a transformation in some physical or chemical property of the solution at the equivalence point

4. An indicator should be used that by a change in physical properties sharply defines the endpoint of the reaction. If there is no visible indicator for the equivalence point detection, the equivalence point can be determined by monitoring the following during the titration: 
- The potential between an indicator electrode and reference electrode (potentiometric titration). The endpoint occurs when the rate of potential change is at a maximum.

- The change in electrical conductivity of the solution (conductometric titration).

- The current which passes through the titration cell between an indicator electrode and a depolarised reference electrode at a suitable applied e.m.f. (amperometric titration)

- The change in absorbance of the solution (spectrophotometric titration)

\subsection{Neutralisation Indicators}

The object of titrating, for example an acid solution with a standard solution of a base, is the determination of the amount of base that is exactly equivalent chemically to the amount of acid present [24]. The point at which this is reached is the equivalence point. An aqueous solution of the corresponding salt results. If both the acid and the base are strong electrolytes, the resultant solution will be neutral and have a $\mathrm{pH}$ of 7 . If either the acid or the base is a weak electrolyte, the salt will be hydrolysed to a certain degree, and the solution at the equivalence point will be slightly alkaline or slightly acidic. For any actual titration the correct endpoint will be characterised by a definite value of the hydrogen ion concentration of the solution, the value depending upon the nature of the acid and base, and the concentration of the solution.

To aid in the determination of these endpoints indicators are used. Acid-base indicators can display a change in colour according to the hydrogen ion concentration of the solution. They change from a predominantly 'acid' colour to a predominantly 'base' 
colour over a small $\mathrm{pH}$ colour-change interval (usually $\pm 2 \mathrm{pH}$ units). This colour-change interval varies depending on the nature of the indicator. For most acid-base titrations there exists an indicator which exhibits a distinct colour change at a $\mathrm{pH}$ close to the equivalence point.

\subsection{Titration/Neutralisation Curves}

Insight into the neutralisation process can be obtained by studying the changes in the $\mathrm{pH}$ during the course of the titration. The most common way of expressing this change is by way of a titration curve $[24,25]$. To construct a titration curve, the titration is done in small equal additions of the titrant. The $\mathrm{pH}$ is monitored after each addition and plotted, as the abscissa (y), against the volume of the base used for the titration, which is plotted as the ordinate (x). The result is a sigmoidal curve, with the centre of the incline indicating the end-point of the titration (Figure 2.1).

Figure 2.1: $\mathrm{pH}$ versus Volume curve indicating an endpoint

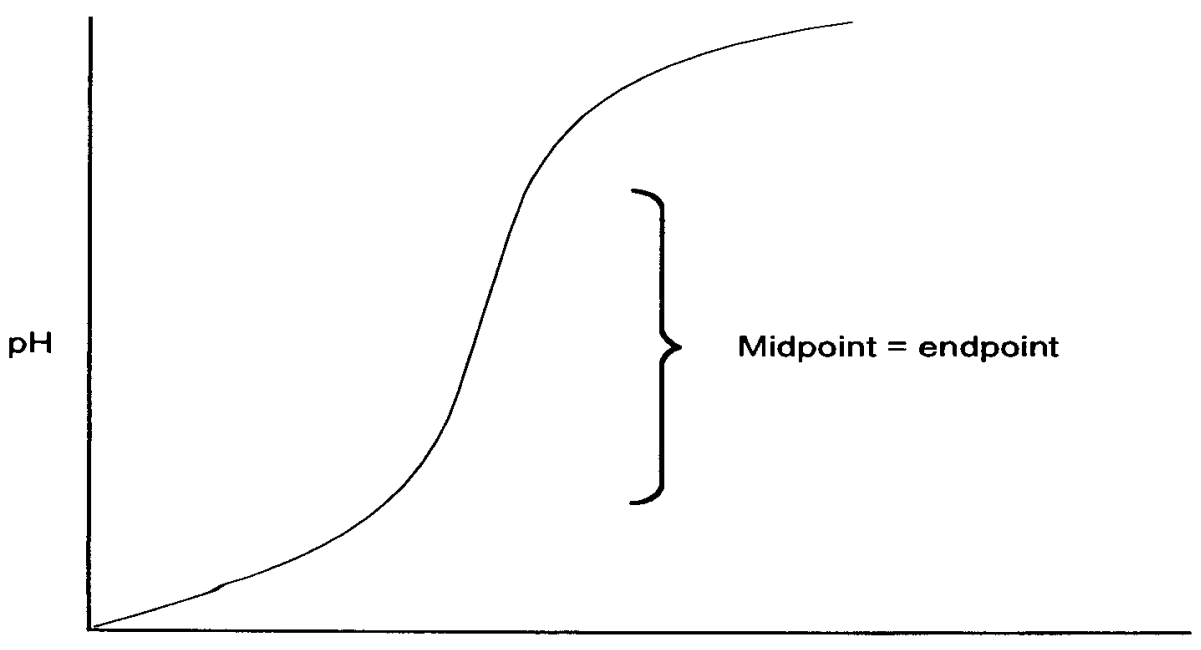

Volume of base added 
In titrations where more than one titratable species is present, more than one incline will result. Each incline describes the endpoint of a particular species in the titration, and these inclines can overlap in very complex matrices. Titration curves are especially useful in complex matrices to determine the contribution of different species to the change in $\mathrm{pH}$.

\subsection{Strong/Weak Acids and Bases [24-33]}

The Lowry-Brфnsted theory of acidity states that in order for a solute to make a solution acidic it must release hydrogen ions to the solution. Strong acids are defined as reagents that ionise completely in water, producing hydrogen ions and corresponding anions (Y) according to eq 2.5 .

$$
\mathrm{HY} \rightarrow \mathrm{H}^{+}+\mathrm{Y}^{-}
$$

In a solution of a strong acid there are assumed to be none of the original undissociated acid molecules, but only hydrogen and the respective anions resulting from the dissociation.

Analogous to the strong acids are strong bases, which dissociate to form hydroxide ions and corresponding cations. Strong bases also ionise completely in solution. 
Acids and bases that are not completely dissociated in solution are termed weak acids or bases. In these acids and bases the reaction to form hydrogen and hydroxide ions occurs only to a small extent. There are many substances which in solution behave as weak acids. They can be classified into the following categories:

1. Molecular species containing an ionisable proton

These include both organic and inorganic acids such as acetic acid and hydrofluoric acid.

2. Anions containing an ionisable proton

Salts such as sodium bisulphate $\left(\mathrm{NaHSO}_{4}\right)$ contain an anion i.e. bisulphate $\left(\mathrm{HSO}_{4}{ }^{-}\right)$which has an ionisable hydrogen.

\section{Cations}

A salt such as ammonium chloride $\left(\mathrm{NH}_{4} \mathrm{Cl}\right)$ is weakly acidic because of the ammonium $\left(\mathrm{NH}_{4}{ }^{+}\right)$cation that ionises to produce a proton. Most metal cations are also considered weak acids. In water these cations become hydrated and produce protons.

\section{Expression for $K_{a}$}

Consider the dissociation of the weak acid HX:

$$
\mathrm{HX}(\mathrm{aq}) \quad \rightleftharpoons \quad \mathrm{H}^{+}(\mathrm{aq})+\mathrm{X}^{-}(\mathrm{aq})
$$

For this equation the equilibrium constant $\mathrm{K}_{\mathrm{a}}$ is formulated as:

$$
\mathrm{K}_{\mathrm{a}}=\left[\mathrm{H}^{+}\right] \cdot\left[\mathrm{X}^{-}\right] /[\mathrm{HX}]
$$


The equilibrium constant, $\mathrm{K}_{\mathrm{a}}$, is called the ionisation constant or dissociation constant of the weak acid HX. The value of the dissociation constant is an indication of the "weakness" of a weak acid. At the $\mathrm{K}_{\mathrm{a}}$ of a weak acid, half of the acid is dissociated.

Expression for $K_{h}$

Similarly to the $\mathrm{K}_{\mathrm{a}}, \mathrm{K}_{\mathrm{h}}$ can be defined to indicate the "acidity" of a cation during hydrolysis. The hydrolysis of a weakly acidic metal ion $\mathrm{M}$ is expressed as:

$\left.\mathrm{M}\left(\mathrm{H}_{2} \mathrm{O}\right)_{\mathrm{n}}{ }^{\mathrm{z}+}+\mathrm{H}_{2} \mathrm{O} \rightleftharpoons \quad \mathrm{M}\left(\mathrm{H}_{2} \mathrm{O}\right)_{\mathrm{n}-\mathrm{l}}(\mathrm{OH})\right\}^{(\mathrm{z}-1)+}+\mathrm{H}_{3} \mathrm{O}^{+}{ }_{(\mathrm{aq})}$

For this equation the equilibrium constant $\mathrm{K}_{\mathrm{h}}$ is formulated as:

$$
\mathrm{K}_{\mathrm{h}}=\left[\left\{\mathrm{M}\left(\mathrm{H}_{2} \mathrm{O}\right)_{\mathrm{n}-1}(\mathrm{OH})\right\}^{(\mathrm{z}-1)+}\right] \cdot\left[\mathrm{H}_{3} \mathrm{O}^{+}\right] /\left[\mathrm{M}\left(\mathrm{H}_{2} \mathrm{O}\right)_{\mathrm{n}}{ }^{\mathrm{z+}}\right]
$$

The equilibrium constant, $\mathrm{K}_{\mathrm{h}}$, is called the hydrolysis constant of the weak metal acid and is an indication of the extent of hydrolysis of the metal cation. 


\subsection{Titration Curves for Polyprotic Acids [24,28,29]}

Suppose that a mixture of two acids, HX and HY, is titrated with standard sodium hydroxide $(\mathrm{NaOH})$. If $\mathrm{HX}$ is much stronger than $\mathrm{HY}$, the first portions of base react almost exclusively with HX, leaving HY unchanged. This process is continued until almost all $\mathrm{HX}$ is converted to $\mathrm{NaX}$. Only when the $\mathrm{HX}$ equivalence point is passed will the added base begin to react with HY.

If there is a great difference in acid strengths (if the ratio of $\mathrm{pK}_{\mathrm{a}}$ 's are 3 or greater), the titration curve shows a sharp equivalence point for $\mathrm{HX}$, and it is possible to titrate $\mathrm{HX}$ in the presence of HY (Figure 2.2a). Also, if the $\mathrm{K}_{\mathrm{h}}$ of $\mathrm{HY}$ is not small the titration may be continued to the endpoint of HY. If the acid dissociation constant for HX is moderately larger than that for $\mathrm{HY}$, an appreciable amount of $\mathrm{HY}$ is converted to $\mathrm{NaY}$ at the equivalence point of $\mathrm{HX}$, and an amount of $\mathrm{HX}$ remains unneutralised. The steepness of the titration curve at the HX equivalence point is reduced, and HX cannot be precisely titrated in the presence of HY,(Figure 2.2b). If HX and HY are of comparable strengths, the $\mathrm{HX}$ equivalence point is obliterated (Figure 2.2c).

A strong acid may be titrated in the presence of an equimolar concentration of a weaker acid with reasonable precision only if the $\mathrm{pK}_{\mathrm{a}}$ of the stronger acid is more than 3 fold greater than that of the weaker [29]. 
Figure 2.2: Titration of a mixture of two acids- The mixture contains $x$ equivalents of $\mathrm{HX}$ and $\mathrm{y}$ equivalents of $\mathrm{HY}$

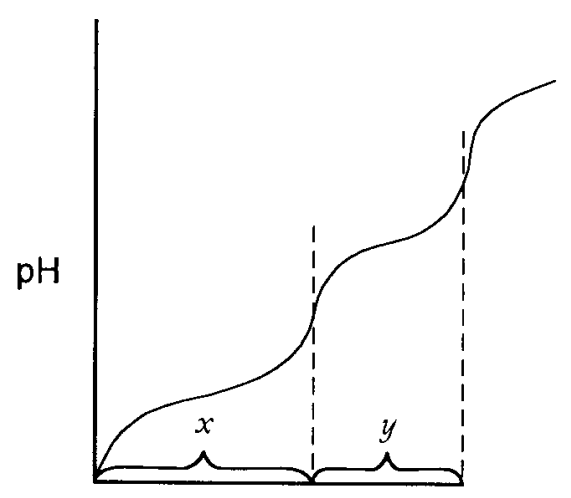

Equivalent of Standard Base

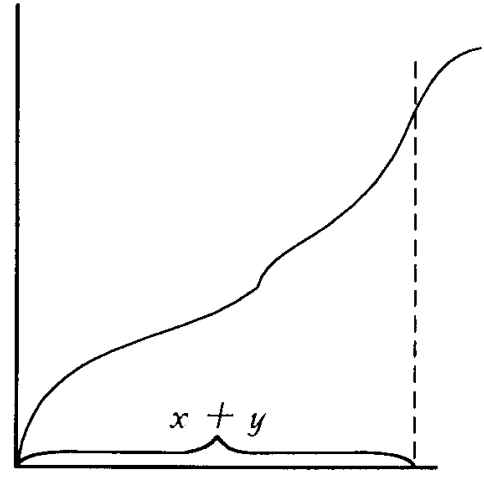

b) $\mathrm{K}_{a, \mathrm{H}_{x}}$ moderately larger than $\mathrm{K}_{a, \mathrm{H}_{y}}$

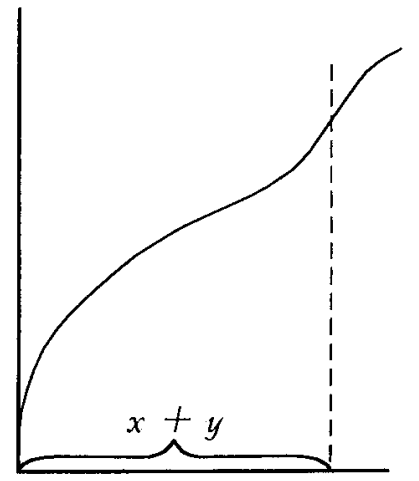

c) $\mathrm{K}_{a, \mathrm{H}_{x}}=\mathrm{K}_{a, \mathrm{H}_{y}}$

The same rule of thumb holds for the hydrolysis constants of hydrolysable cationic species. The first proton of sulphuric acid is almost completely dissociated in aqueous solutions. The resultant bisulphate ion acts as a weak acid and the second proton is not completely dissociated. The $\mathrm{pK}_{\mathrm{a}}$ of the bisulphate ion $\left(\mathrm{HSO}_{4}{ }^{-}\right)$is $1.9[13,14,27]$. Since the $\mathrm{pK}_{\mathrm{h}}$ of the trivalent iron cation is 2.2 [27], the difference between these two values is very small. At a pH of 2.2 [27], half of the iron(III) have undergone first hydrolysis, whereas the bisulphate ions have not completely been neutralised. This explains the difficulties in determining the individual concentrations of these two species in solution with a free acid titration. On a pH vs. titrant volume curve this translates into two inclines merged into the background curve. It makes endpoint allocation to individual species very difficult and in some cases impossible. 


\subsection{Acid-Base Concepts [24-33]}

The acid-base 'theories' are really definitions. They offer an insight into what is most convenient in a particular situation as opposed to what is 'right'. All the definitions are compatible and have a basic parallelism.

\section{Lowry- Bronsted Definition}

This concept defines acids as proton donors and bases as proton acceptors (equation 2.10). For aqueous solutions this definition does not significantly differ from the Arrhenius definition of hydrogen ions(acids) and hydroxide ions(bases).

$$
\begin{aligned}
& 2 \mathrm{H}_{2} \mathrm{O} \rightleftharpoons \quad \mathrm{H}_{3} \mathrm{O}^{+}+\mathrm{OH}^{-} \\
& \text {Pure solvent } \\
& \quad \text { acid base }
\end{aligned}
$$

This concept is useful since it is able to handle any protonic solvent as well as reactions which are acid-base in character but not necessarily neutralisation reactions. The emphasis placed by the Bronsted-Lowry concept on competition for protons is a limiting factor since it is only useful in a protonic solvent system. Other acid-base definitions were formulated in an attempt to extend the concept to systems not containing protons. For much of this study this is the preferred definition since most of the reactions involve proton donors and acceptors. 


\section{Lux-Flood definition}

This definition describes acid-base behaviour in terms of the oxide ion. This concept was proposed to treat non-protonic systems. According to this definition the base is an oxide donor and the acid is an oxide acceptor and for this reason the Lux-Flood definition is limited to systems such as molten oxides.

\section{Solvent System Definition}

Many solvents auto-ionise with the formation of a cationic and an anionic species as does water:

$$
\begin{aligned}
& 2 \mathrm{H}_{2} \mathrm{O} \rightleftharpoons \mathrm{H}_{3} \mathrm{O}^{+}+\mathrm{OH}^{-} \\
& 2 \mathrm{H}_{2} \mathrm{SO}_{4} \rightleftharpoons \quad \mathrm{H}_{3} \mathrm{SO}_{4}^{+}+\mathrm{HSO}_{4}^{-}
\end{aligned}
$$

For treatment of acid-base reactions, particularly neutralisations, it is convenient to define the acid as a species that increases the concentration of the characteristic cation of the solvent and the base as a species that increases the concentration of the characteristic anion. The advantage of this definition is purely for convenience. The definition can also be used for non-aqueous solvents. However, it has been criticised for concentrating too heavily on ionic reactions in solution and on the chemical properties of the solvent to the neglect of the physical properties. When the ionic species formed in solution are known 
the solvent system approach can be useful. In solvents that do not form ions and if little or nothing is known of the ionic species the definition is of no use.

\section{Lewis Definition}

Here acid-base behaviour is defined in terms of electron pair donation and acceptance. This definition is most widely used due to its simplicity and wide applicability. A base is defined as an electron-pair donor and an acid as an electron-pair acceptor. The definition includes reactions discussed by the other definitions but also reactions in which no ions are formed and no hydrogen or other ions are transferred. This definition includes all reactions involving hydrogen ion, oxide ion, or solvent interactions, as well as acid-base adducts and all co-ordination compounds.

\section{Usanovich Definition}

All reactions of Lewis acids and bases are included and this definition extends the Lewis concept by removing the restriction that the donation or acceptance of electrons be as shared pairs. The definition is: An acid is any chemical species which reacts with bases, gives up cations, or accepts anions or electrons, and, conversely, a base is any chemical species which reacts with acids, give up anions or electrons, or combines with cations. The definition therefore includes all Lewis acid-base reactions and in addition includes redox reactions. 


\subsection{Hard and Soft Acids and Bases [24-34]}

Metal ions can displace one another from a complex because it forms a stronger bond with the ligand. Regardless of the complexing ion the displacement of cations follow a certain trend related to the stability of metal complexes. An understanding of the order of stability of metal complexes can aid in the masking of to prevent competition for water molecules and hence prevent hydrolysis of the hydrolysable cations.

The Irving-Williams series describes this trend of the stability of metal complexes. For a certain ligand, the stability of the complex with dipositive metal ions follows a certain order i.e.

$\mathrm{Ba}^{2+}<\mathrm{Sr}^{2+}<\mathrm{Ca}^{2+}<\mathrm{Mg}^{2+}<\mathrm{Mn}^{2+}<\mathrm{Fe}^{2+}<\mathrm{Co}^{2+}<\mathrm{Ni}^{2+}<\mathrm{Cu}^{2+}<\mathrm{Zn}^{2+}$

This series indicates that for a particular ligand the order of stability is left to right i.e. metals to the right in the series form more stable complexes with a particular ligand than metals to the left. The stability of complexes increases from $\mathrm{Ba}^{2+}$ to $\mathrm{Zn}^{2+}$. This order also correlates with the decrease in size across the series and to a degree to ligand field effects.

It has also been observed that certain ligands form their most stable complexes with metal ions such as silver and mercury(II) whilst other ligands prefer aluminium(III), titanium(IV), cobalt(III). Ligands and metal ions were classified as belonging to type (a) or (b) according to their preference for bonding. Class (a) metal ions include alkali metals, alkaline earth metals, and lighter transition metals, and those in higher oxidation 
states such as titanium(IV), chromium(III), iron(III), cobalt(III) and the hydrogen ion. Class (b) metal ions include those of the heavier transition metals and those in lower oxidation states such as copper(I), silver(I), mercury(I), palladium(II), and platinum(II). Ligands are classified as type (a) or (b) according to their preferences toward either of the above classes. Pearson $[20,25,27,34]$ has suggested the terms 'hard' to describe the members of class (a) and 'soft' to describe class (b). Therefore a hard acid is a type (a) metal ion and a hard base is a ligand that forms a stable compound with the hard acid. A soft acid is a type (b) metal ion and a soft base is a ligand that binds stably to the acid. Hard species, both acids and bases, tend to be small, slightly polarisable species and soft acids and bases tend to be larger and more polarisable. Pearson suggested this simple rule of thumb- 'Hard acids prefer to bind to hard bases and soft acids prefer to bind to soft bases'.

There are tables available which list hard and soft acids and bases. It is important to remember that the terms hard and soft are relative and that there is no sharp dividing line. Even within a group of hard or soft, not all have equivalent hardness or softness. 


\subsection{Acid-Base Strength and Hardness and Softness}

Acid and base strengths should be distinguished from hardness and softness which refers to the stability of hard-hard and soft-soft interactions [24-33]. It is possible for a strong acid or base to displace a weaker one, even though this appears to violate the principle of hard and soft acids and bases. When considering acid-base interactions, it is necessary to consider both strength and hardness-softness. The Irving-Williams series shows the importance of inherent acidity and a hard-soft factor. The series of increasing stability from $\mathrm{Ba}^{2+}$ to $\mathrm{Cu}^{2+}$ is an indication of acidity of the metal. Also there is an overlapping hard-soft factor in which the softer species occurring later in the series favour ligands $\mathrm{S}>\mathrm{N}>\mathrm{O}$. The harder alkaline earth and early transition metal ions preferentially bind $\mathrm{O}>\mathrm{N}>\mathrm{S}$.

\subsection{Potentiometry [24,35]}

When a metal $\mathrm{M}$ is immersed in a solution containing its own ion $\mathrm{M}^{\mathrm{n}+}$, then an electrode potential is established, the value of which is given by the Nernst equation:

$$
E=E^{\theta}+(R T / n F) \ln a_{M n+}
$$

where $\mathrm{E}^{\theta}$ is a constant, the standard electrode potential of the metal. $\mathrm{E}$ can be measured by combining the electrode with a reference electrode, and measuring the e.m.f. of the resultant cell. If $E_{r}$ of the reference electrode is known, the value of the electrode 
potential can be deduced, and if $E^{\theta}$ the standard electrode potential of the given metal is known, the metal ion activity $\mathrm{a}_{\mathrm{Mn}}$ in the solution can then be calculated. For dilute solutions the measured ionic activity will be virtually the same as the ionic concentration, and for stronger solutions, given the value of the activity coefficient, the measured ionic activity can be converted into the corresponding concentration.

The use of a single measurement of electrode potential to determine the concentration of an ionic species in solution is referred to as direct potentiometry. From direct potentiometry arose the potentiometric titration. It is a titration in which potentiometric measurements are carried out in order to fix the endpoint. The method is concerned with changes in electrode potential are monitored rather than an accurate value for the electrode potential. In such a titration, the change in cell e.m.f. occurs most rapidly in the neighbourhood of the endpoint, and various methods can be used to ascertain the endpoint at which the rate of potential change is at a maximum, which is the endpoint of the titration.

\subsection{Relation between Potential and pH [36]}

From the Sörensen definition of $\mathrm{pH}$ given earlier:

$$
\mathrm{pH} \equiv-\log _{10}\left[\mathrm{H}^{+}\right]
$$

Since concentration is linked to activity by:

$$
\begin{aligned}
& \mathrm{a}_{\mathrm{H}+}=\gamma \mathrm{c}_{\mathrm{H}+} \\
& \mathrm{pH} \equiv-\log _{10} \mathrm{a}_{\mathrm{H}+}
\end{aligned}
$$


Substituting this into the Nernst equation we get:

$$
\mathrm{E}=\mathrm{E}^{\theta}-(2.303 \mathrm{RT} / \mathrm{nF}) \mathrm{pH}
$$

Hence using a suitable $\mathrm{pH}$ electrode and a reference electrode the potential of the solution can be related to $\mathrm{pH}$ which can be monitored during the titration in order to detect the endpoint.

\subsection{Automatic Potentiometric Titrations}

Automatic potentiometric titrations allow for the production of the titration curve relating to the potentiometric titration under investigation. The titrant is delivered from a burette linked to the instrument. The $\mathrm{pH}$ and volume are recorded and a curve is printed at the end of the titration indicating the endpoints.

\subsection{Endpoint Location [24]}

A titration curve has a sigmoidal shape and the endpoint will be located in the centre of the steeply rising portion of the curve, the point of inflection. When the curve shows a very clearly marked incline, an approximate value of the endpoint can be given as being mid-way along the incline of the curve. To fix the endpoint exactly a geometric construction is necessary. Four methods may be adopted:

1. Method of bisection

2. Method of parallel tangents

3. Method of circle fitting 
4. Derivative method

1. The method of bisection is used when there are reasonably good straight lines before and after the incline of the curve. Each of these straight lines are extended to the right and left respectively, and then at suitable points vertical lines are constructed, one to the right of the incline of the titration curve and one to the left (Figure 2.3). These vertical lines are then bisected, and the midpoints joined. The endpoint of the titrations taken where this line cuts the titration curve.

Figure 2.3: Bisection Method of endpoint location

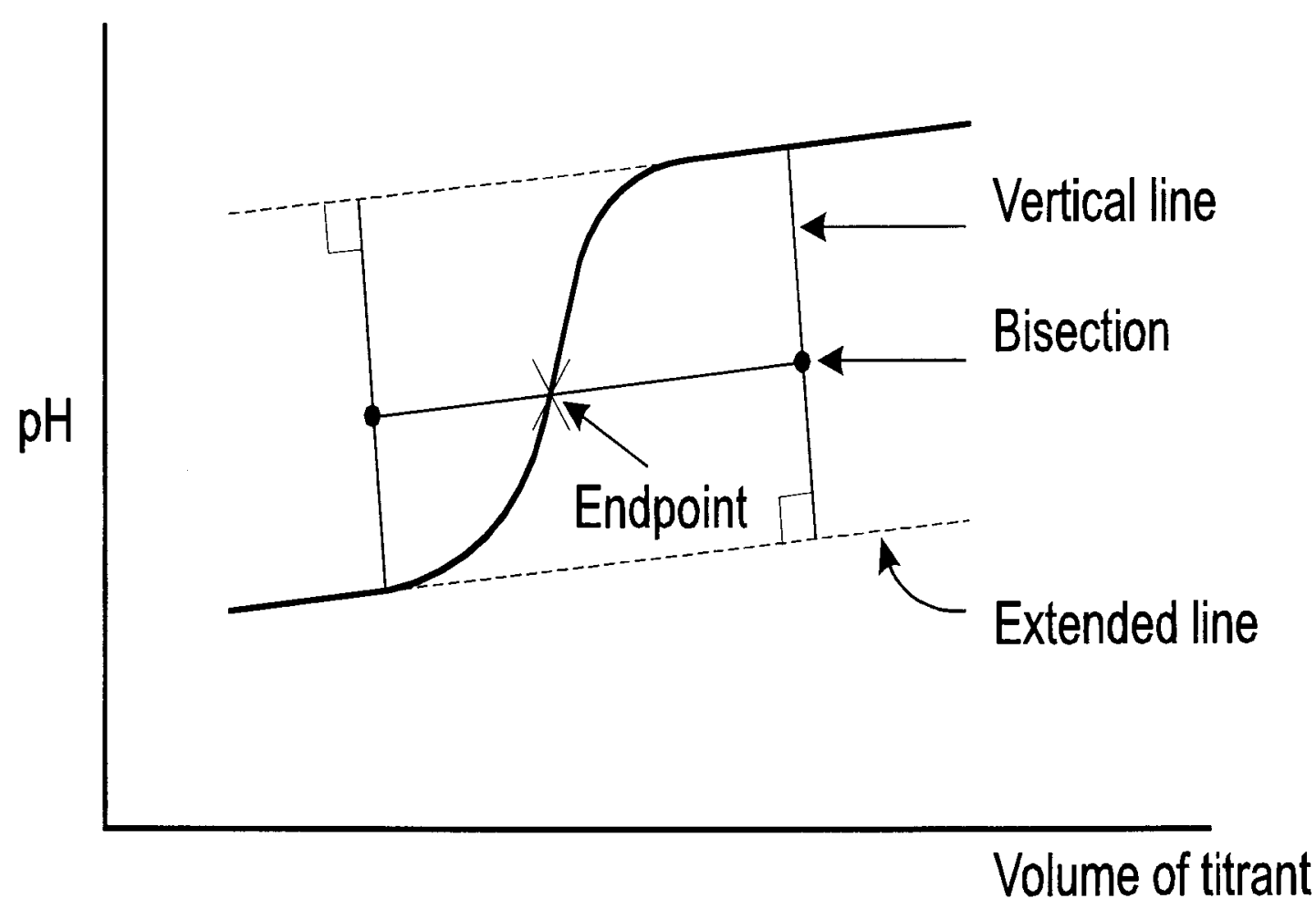


2. In the method of parallel tangents, parallel lines are constructed on the titration curve starting with a line tangential to the upper and lower points of the curve. A parallel line equidistant from the tangential lines is then constructed. The intersection of this parallel line with the incline is the endpoint (Figure 2.4).

Figure 2.4: Method of parallel tangents for endpoint location

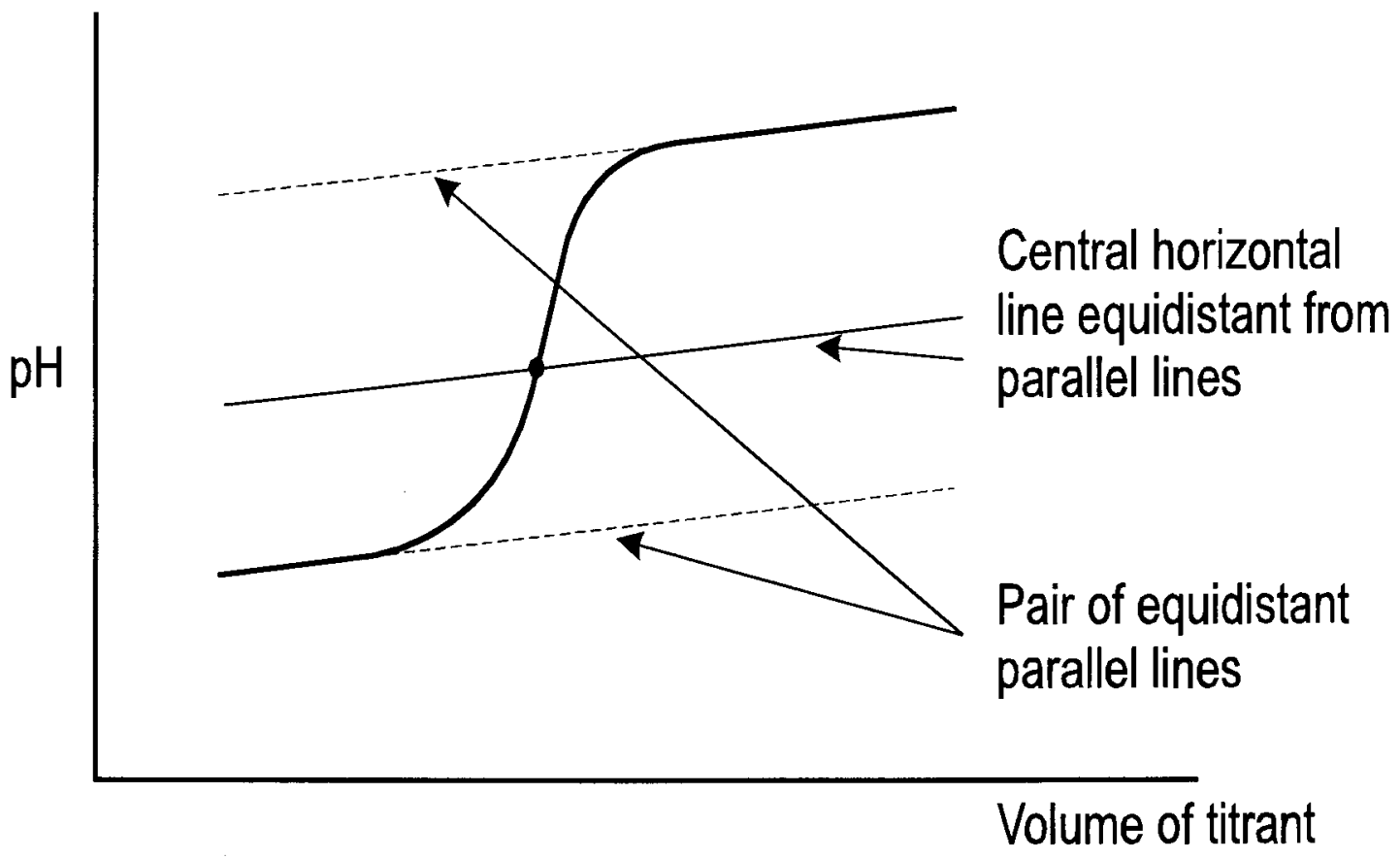

3. In the method of circle fitting a circle is drawn to fit one of the bends of the curve (Figure2.5). The centre of this circle should be marked. A second circle is drawn to fit the other bend of the curve and the centre marked. Draw a line to join the two centres. Where this line intersects the curve is where the endpoint is. 
Figure 2.5: Method of circle fitting for endpoint location

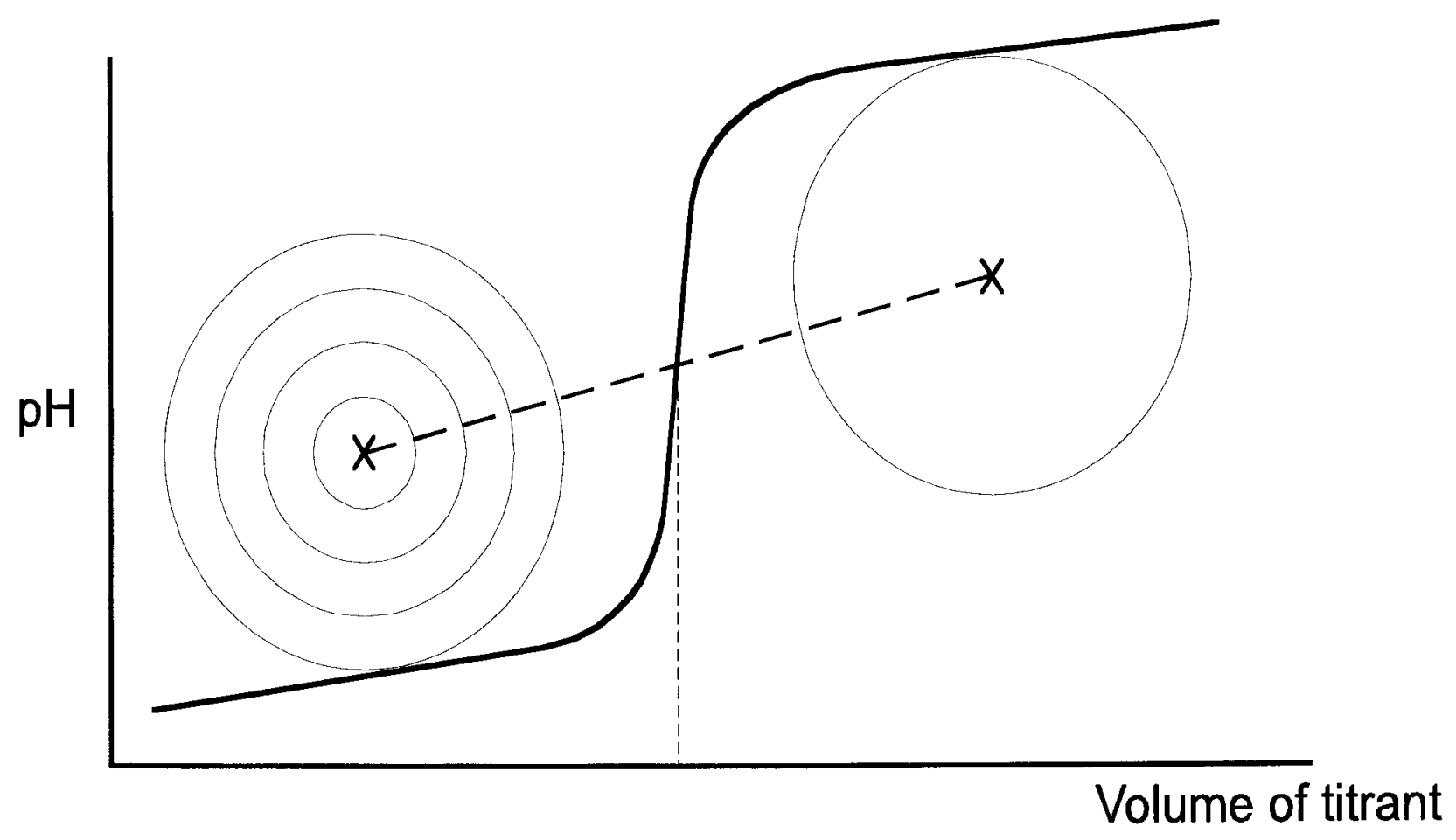

4. The derivative methods are a more accurate method. These methods involve the plot of the first derivative $\Delta \mathrm{pH} / \Delta \mathrm{V}$ vs. $\mathrm{V}$ where the peak of the graph indicates the endpoint. The second derivative curve $\Delta^{2} \mathrm{pH} / \Delta \mathrm{V}^{2}$ vs. $\mathrm{V}$ can also be plotted, here the midpoint between the peak and the dip is the endpoint. 
Figure 2.6: Derivative method of endpoint location

\section{$x=$ Endpoint}
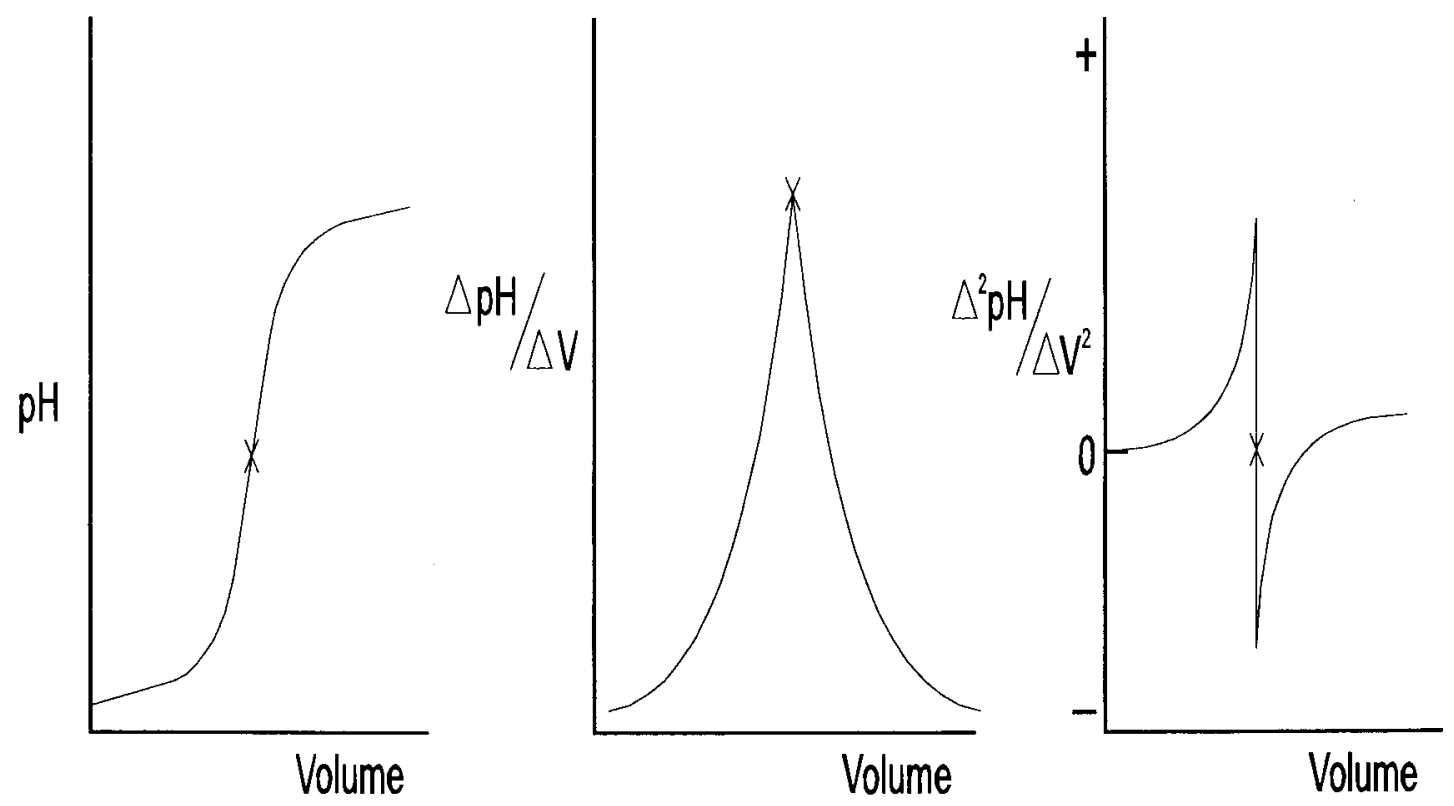


\subsection{Automatic Titrators $[20,21,27,30]$}

A titration system usually consists of the following:

1. Titrant delivery system

2. Titration vessel

3. Titration monitor

4. Controlling device

Figure 2.7: Components of an autotitrator

\section{Iitrant Delivery System}

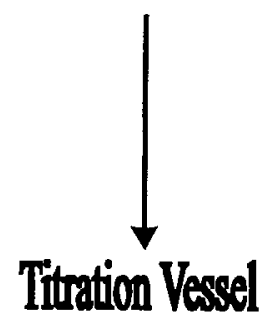

1. Titrant delivery system

The most common automatic titrators used in the lab are auto burettes, which can be classified as:

a) Burettes with automatic valves

b) Auto syringe burettes

c) Other types of auto burette 
a) The typical burette with an automatic stopcock resembles those used in classical titrimetric analysis - a long, thin glass tube with a volume scale, equipped with a valve that operates automatically.

b) Modern auto titrators usually include syringe (piston or plunger) burettes from which the titrant is dispersed at a pre-set rate. The tube resembles a medical syringe and is made of glass or a rigid plastic material to a uniform diameter. The plunger, made of glass, corrosion resistant metal or, preferably PTFE, is also manufactured to a high precision so as to move freely inside the tube and yet provide a leak-proof seal. One outlet of the burette is thus connected to the titrant reservoir and the other leads into the solution to be titrated.

c) Constant - flow burettes are also sometimes used. These overcome the problem of non-uniform rate of delivery arising from the use of ordinary burettes by keeping the hydrostatic pressure constant.

\section{Titration Vessel}

The characteristics of the vessel where the sample and titrant is mixed depend to a great extent on those of the monitoring system used. A simple beaker where the probe and delivery tube can be immersed is usually all that is required.

\section{Titration Monitor}

The most convenient way to monitor titrations is electroanalytically. Potentiometric titrations are the most widely used in this respect, although, colorimetric detectors can also be applied. 
Degree of Automation

There are two levels of automatic titrators:

a) Hardware-controlled titrators

In auto titrators, reagent dispensing is frequently controlled in a simple manner by use of detector signal feedback. One of the simplest and commonest methods of hastening the titration is the anticipation principle, based on a present anticipation detector signal level. The reagent is added at a relatively rapid rate until the measured signal attains the pre-set level, from which the rate is decreased. In this way, the titration rate is increased with no significant detriment to the precision.

b) Micro-processor controlled titrators

The Metrohm autotitrator is furnished with data storage facilities for storing the titration methods most frequently used together with other information. All operations related to data entry, sequence control, calculation, data output etc. can be handled. The programs for titration control, evaluation methods, and standard parameter sets can be stored. The Metrohm autotitrator allows for various entries. Printers, balances and dialogue language can be selected. It allows for the input of calculation formula and constants. The data output can be defined in terms of what information should be printed i.e. type of graphs etc. Various parameters for the control of the titration can also be selected. 


\section{Chapter 3: Experimental}

\subsection{Apparatus}

All the titrations were performed with a Metrohm automatic titrator, model 716 DMS

Titrino, which was connected to an Epson LX-300 printer. The titrant was delivered from a $50 \mathrm{ml}$ syringe burette to a beaker containing the analyte solution. The solution was stirred magnetically. A combined $\mathrm{pH}$ and reference electrode, with a $3 \mathrm{~mol} / \mathrm{l}$ potassium chloride bridging solution, was used throughout the investigation as the potentiometric probe. The titrator was microprocessor controlled. Various parameters had to be selected during the set up of the titration method.

The titration parameters were set as follows: The measuring point density was set to the recommended value 4 . Values allowed are between $0-9$. A low value indicates small volume increments. and implies a curve with fine details. The disadvantage of this is that even signal noise could lead to unwanted equivalence points. The minimum increment is the smallest volume of titrant that is added at the beginning of the titration and at the equivalence point of the titration. This was set to $40 \mu 1$. Use of smaller values also leads to unwanted equivalence points. The rate of titration is the rate of titrant addition outside the control range and set to maximum to enhance the analysis time. The signal drift was set to $1 \mathrm{mV} / \mathrm{min}$ ensuring that measured values are acquired after the drift has settled to less than $1 \mathrm{mV} / \mathrm{min}$. Equilibration time, which is the time that the analyser pauses to allow the solution to reach equilibrium before the next addition of titrant, was set to $10 \mathrm{~s}$. The 
pause time was set to 10 s to allow the solution time to mix properly before titrant addition commences. The evaluation parameter was set to endpoint recognition to allow for multiple endpoints to be reported.

The titration curve and its first derivative was plotted by the auto-titrator during this investigation. The endpoints detected by the auto titrator were not always clear cut as small changes were sometimes given as endpoints, and the 'correct' endpoints often had to be identified by the analyst. In this investigation it was decided to use the titration curve together with the method of parallel tangents to determine the endpoints.

\subsection{Reagents}

All the reagents used were of analytical-reagent grade except where specified. Distilled water or methanol was used to prepare both reagents and samples. Standard solutions of sulphuric acid were prepared from concentrated sulphuric acid specific gravity $1.84 \mathrm{~g} / \mathrm{ml}$, and standardised with $0.1 \mathrm{~mol} / 1$ sodium carbonate or $0.1 \mathrm{~mol} / \mathrm{l}$ sodium hydroxide. The sodium hydroxide was standardised with potassium hydrogen phthalate (dried at $110^{\circ} \mathrm{C}$ for 1 hour) and bromothymol blue indicator $(0.2 \%)$. Bromothymol blue indicator was prepared by mixing $3 \mathrm{ml}$ of $0.1 \mathrm{~mol} / 1$ sodium hydroxide with $0.2 \mathrm{~g}$ of the solid indicator and diluting the solution to $100 \mathrm{ml}$ with stirring. Saturated aqueous solutions of lithium chloride, sodium chloride, calcium chloride, zinc chloride, and magnesium chloride were used. Where specified, saturated methanol solutions of these salts were used. Iron(II) and copper solutions were prepared from the sulphate salt, the iron(III) was prepared from the 
nitrate salt. The cobalt and manganese solutions were prepared from the salts as stated in the results. Depending on the hydrolysable cations and acid concentrations, different sample aliquots were transferred into a beaker. Fifty millilitres of the reagent to be tested was added and the sample was titrated with $0.1 \mathrm{~mol} / \mathrm{l}$ sodium hydroxide to a $\mathrm{pH}$ value of approximately 8 . The point of equivalence was determined from the inflection point(s) on the titration curve, and the acid concentration was calculated accordingly. 


\section{Chapter 4: Results and Discussion}

\subsection{Establishing Interferences}

\subsubsection{Effect of Copper(II), Nickel(II) and Cobalt(II) on Acid}

\section{Determinations.}

A solution that contained $53 \mathrm{~g} / 1$ of copper(II) sulphate was spiked with $4 \mathrm{~g} / 1$ of sulphuric acid and analysed for free acid content. The presence of copper(II) in solutions had no significant effect on the free acid concentration, even when the ratio of copper(II) to sulphuric acid was as high as 1: 0.069. This observation could be explained by the $\mathrm{pH}$ of hydrolysis $\left(\mathrm{pK}_{\mathrm{h}}\right.$ ) of copper(II) which was at $\mathrm{pH} 6$ [32]. The copper(II) hydrolysis occurs after the $\mathrm{pH}$ of neutralisation of the bisulphate at $\mathrm{pH} 3.25$ [32].

The results obtained for acid determination in the presence of $127 \mathrm{~g} / 1$ cobalt(II) nitrate in the presence of $4 \mathrm{~g} / \mathrm{l}$ of sulphuric acid are consistent with the $\mathrm{pH}$ of hydrolysis of cobalt(II) $(\mathrm{pH}=8)$ [32]. The $\mathrm{pH}$ of neutralisation occurs before the endpoint of hydolysis of cobalt(II) and hence no interference from cobalt(II) hydrolysis is seen. Cobalt(II) nitrate, sulphate, and chloride were titrated to check the effect of the anions on the acid determination. The three anions did not have a significant effect on the acid results.

A solution of $171 \mathrm{~g} / \mathrm{l}$ nickel(II) nitrate and $4 \mathrm{~g} / \mathrm{l}$ sulphuric acid does not have a significant effect on the acid determination since the $\mathrm{pH}$ of hydrolysis of nickel(II) is 8 [32]. 
Titration of solutions that contain high concentrations of copper, cobalt, and nickel solutions should be done by auto titration or with a $\mathrm{pH}$ meter since the solutions are highly coloured and would distort the endpoint of an indicator titration.

\subsubsection{Effect of Iron(II) on Acid Determination}

Since iron(II) hydroxide precipitates at a $\mathrm{pH}$ of 6 [32], it would be expected that it would have no effect on the free acid concentration. Table 4.1 however indicated that the results were high and erratic.

The accuracy of the determinations for samples containing $38 \mathrm{~g} / \mathrm{l}$ iron(II) sulphate was poor as shown in Table 4.2. This was probably due to the presence of iron(III) which resulted from the oxidation of iron(II) by air as indicated by equation 4.1 showing that iron(III) is produced and protons are consumed, both of which adversely affect the alkali titration [7].

$$
2 \mathrm{Fe}^{2+}+(\mathrm{O})+2 \mathrm{H}^{+} \quad \rightarrow \quad 2 \mathrm{Fe}^{3+}+\mathrm{H}_{2} \mathrm{O}
$$

The results imply that sampling, handling and analysis of solutions that contain iron(II) should be done under anaerobic conditions. Alternatively analysis should be done as soon as the sample is received in order to minimise the effects of oxidation. 
Table 4.1: Determination of sulphuric acid in the presence of iron(II)

\begin{tabular}{|l|l|l|l||}
\hline \hline$(\mathrm{H} / \mathrm{H})$ & {$\left[\mathrm{SO}_{4}\right]$ added } & {$\left[\mathrm{Fe}^{2+}\right]$ added } & {$\left[\mathrm{H}_{2} \mathrm{SO}_{4}\right]$ determined } \\
$(\mathrm{g} / \mathrm{l})$ & $\%$ Error \\
\hline 4.12 & 0.0 & 4.12 & \\
\hline 4.12 & 0.1 & 4.17 & 1.21 \\
\hline 4.12 & 0.5 & 4.32 & 4.85 \\
\hline 4.12 & 1.0 & 4.41 & 7.04 \\
\hline 4.12 & 1.5 & 4.41 & 7.04 \\
\hline 4.12 & 2.0 & 4.36 & 5.83 \\
\hline 4.12 & 3.0 & 4.36 & 5.83 \\
\hline 4.12 & 4.0 & 4.30 & 4.37 \\
\hline 4.12 & 5.0 & 4.41 & 7.04 \\
\hline 160.0 & 30 & 147.98 & \\
\hline
\end{tabular}


Table 4.2: Accuracy of sulphuric acid determinations in the presence of iron(II).

\begin{tabular}{|l|l|l||}
\hline$\left[\mathrm{Fe}^{2+}\right],\left[\mathrm{H}_{2} \mathrm{SO}_{4}\right]$ added $(\mathrm{g} / \mathrm{l})$ & {$\left[\mathrm{H}_{2} \mathrm{SO}_{4}\right]$ determined $(\mathrm{g} / \mathbf{l})$} & \% Error \\
\hline $5.0 \mathrm{Fe}^{2+}, 4.12 \mathrm{H}_{2} \mathrm{SO}_{4}$ & 4.32 & 4.85 \\
\hline $5.0 \mathrm{Fe}^{2+}, 4.12 \mathrm{H}_{2} \mathrm{SO}_{4}$ & 4.41 & 7.04 \\
\hline $5.0 \mathrm{Fe}^{2+}, 4.12 \mathrm{H}_{2} \mathrm{SO}_{4}$ & 4.36 & 5.83 \\
\hline $5.0 \mathrm{Fe}^{2+}, 4.12 \mathrm{H}_{2} \mathrm{SO}_{4}$ & 4.71 & 14.32 \\
\hline $5.0 \mathrm{Fe}^{2+}, 4.12 \mathrm{H}_{2} \mathrm{SO}_{4}$ & 4.55 & 10.44 \\
\hline $5.0 \mathrm{Fe}^{2+}, 4.12 \mathrm{H}_{2} \mathrm{SO}_{4}$ & 4.81 & 16.75 \\
\hline $0 \mathrm{Fe}^{2+}, 4.12 \mathrm{H}_{2} \mathrm{SO}_{4}$ & 4.12 & 0 \\
\hline $0 \mathrm{Fe}^{2+}, 4.12 \mathrm{H}_{2} \mathrm{SO}_{4}$ & 4.12 & 0 \\
\hline $0 \mathrm{Fe}^{2+}, 4.12 \mathrm{H}_{2} \mathrm{SO}_{4}$ & 4.12 & 0 \\
\hline $0 \mathrm{Fe}^{2+}, 4.12 \mathrm{H}_{2} \mathrm{SO}_{4}$ & 4.12 & 0 \\
\hline \hline
\end{tabular}

\subsubsection{Effects of Manganese(II) on Acid Determination}

The results for the acid determination in the presence of manganese(II) was significantly higher than the actual acid value (Table 4.3). During the titration the sample turned from a colourless solution to light yellow at $\mathrm{pH} \sim 2$. The yellow colouring cannot be due to manganese(II) hydrolysis because this occurs at a $\mathrm{pH}$ of 9 [32] and, in addition, manganese hydroxide is not a yellow precipitate. It was thought that the colouring could be due to iron, but according to specification on the reagent bottle the iron concentration in manganese nitrate $\left(\mathrm{Mn}\left(\mathrm{NO}_{3}\right)_{2}\right)$ is lower than in the other cation salts used. To discount 
a possible anion effect manganese chloride $\left(\mathrm{MnCl}_{2}\right)$ and manganese sulphate $\left(\mathrm{MnSO}_{4}\right)$ were chosen to determine the effect of the anions. Manganese chloride gave results that were significantly closer to the actual acid value. Manganese sulphate forms a milky solution and hence the solution concentration could not be determined with any confidence. Manganese nitrate and manganese chloride behave differently in terms of their interference effect on the acid determination even when the manganese(II) concentration is the same. An explanation for this is that the $\mathrm{NO}_{3}{ }^{-}$undergoes reduction to $\mathrm{NO}_{2}{ }^{-}$(equation 4.2) [22]. This reduction explains the yellow colour observed in the solution when the titration is started. $\mathrm{The}^{2+}$ therefore undergoes oxidation in the presence of hydroxide (equation 4.3) [22]. The consumption of hydroxide accounts for the high titres.

$$
\begin{aligned}
& \mathrm{NO}_{3}{ }^{-}+\mathrm{H}_{2} \mathrm{O}+2 \mathrm{e}^{-} \rightleftharpoons \quad \mathrm{NO}_{2}{ }^{-}+2 \mathrm{OH}^{-} \quad 0.01 \mathrm{~V} \\
& \mathrm{Mn}(\mathrm{OH})_{2}+\mathrm{OH}^{-} \rightleftharpoons \quad \mathrm{Mn}(\mathrm{OH})_{3}+\mathrm{e}^{-} \quad 0.40 \mathrm{~V}
\end{aligned}
$$

The manganese chloride behaves differently because there is no spontaneous reduction reaction for the chloride that would drive the oxidation of the manganese(II). Hence manganese chloride does not undergo a redox reaction whereas manganese nitrate does. 
Table 4.3: Determination of sulphuric acid in the presence of $\mathrm{Mn}(\mathrm{II})$

\begin{tabular}{||l|l|l|l||}
\hline \hline $\left.\mathbf{M n}^{2+}\right]$ added $(\mathbf{g} / \mathbf{l})$ & {$\left[\mathrm{H}_{2} \mathbf{S O}_{4}\right]$ added $(\mathbf{g} / \mathbf{l})$} & {$\left[\mathbf{H}_{2} \mathbf{S O}_{4}\right]$ determined $(\mathrm{g} / \mathbf{l})$} & \% Error \\
\hline $233.2 \mathrm{Mn}\left(\mathrm{NO}_{3}\right)_{2}$ & 4.7 & 5.30 & 12.76 \\
\hline $233.2 \mathrm{Mn}\left(\mathrm{NO}_{3}\right)_{2}$ & 4.7 & 5.37 & 14.25 \\
\hline $204.1 \mathrm{Mn}\left(\mathrm{NO}_{3}\right)_{2}$ & 4.7 & 5.14 & 9.4 \\
\hline $197.3 \mathrm{MnCl}_{2}$ & 4.7 & 4.68 & 0.42 \\
\hline $221.9 \mathrm{MnCl}_{2}$ & 4.7 & 4.87 & 3.64 \\
\hline
\end{tabular}

\subsubsection{Effect of Iron(III) on Acid Determination}

Titration of acid in the presence of $90 \mathrm{~g} / \mathrm{l}$ iron(III) nitrate causes major interferences. Precipitation is seen even before the acid endpoint is reached. The $\mathrm{pK}_{\mathrm{h}}$ of iron (III) is 3 [32] and this coincides with the $\mathrm{pH}$ of acid neutralisation for the bisulphate. For samples containing iron(III) alone the potassium iodide method in use at MINTEK proves accurate for acid determination.

\subsubsection{Conclusion}

Solutions of copper(II), nickel(II), cobalt(II), iron(II), manganese(II), and iron(III) were spiked with sulphuric acid and the free acid concentration was determined. Only iron(III) and manganese(II) cause notable interference. Manganese nitrate causes interference on the acid determination because the nitrate undergoes reduction reaction spontaneously 
which drives the oxidation of the manganese(II). The chloride in manganese chloride does not undergo reduction and hence manganese chloride does not affect the free acid determinations significantly. Acid determination in the presence of copper(II), cobalt(II), and nickel(II) present no real problems. A potentiometric auto-titration should be used instead of an indicator for highly coloured sample solutions to prevent the endpoint colour being distorted. Care must be taken with solutions containing iron(II) to ensure that no oxidation occurs prior to titration. 


\subsection{Effect of Complexing Agents}

During the determination of free acid in the presence of hydrolysable cations a method was suggested to remove the interfering cation. The cation could be removed by using a complex forming agent to mask the cation. Potassium oxalate, tartrate and fluoride were suggested [4].

\subsubsection{Potassium Oxalate as Complexing Agent}

Potassium oxalate addition was extensively researched $[4,11,12]$. It was used for acid determination in different sample types. This method is based on the formation of complexes between the cations present in the solution and the potassium oxalate. The oxalate ions form stable complexes with the cations particularly the iron(III). When the free acid is titrated with sodium hydroxide, the stability of these complexes prevents hydrolysis at the $\mathrm{pH}$ value of the neutral point. This method was adapted for use at MINTEK laboratories.

Table 4.4 depicts results that were obtained for solutions containing sulphuric acid and varying amounts of iron(II), iron(III), and copper(II) using the potassium oxalate as the complex forming reagent. The results showed that the potassium oxalate method gives different results for acid determination, although the difference would indicate that the accuracy was good. In solutions with iron(III) the accuracy decreased and the precision 
was erratic. Also the precipitation of ions started before the neutral point was reached.

For these reasons potassium oxalate was not used as a complexing agent.

Table 4.4: The effect of varying amounts of iron and copper(II) on the potassium oxalate method

\begin{tabular}{|c|c|c|c|c|}
\hline $\begin{array}{l}{\left[\mathrm{H}_{2} \mathrm{SO}_{4}\right]:\left[\mathrm{Fe}^{2+}\right]:} \\
{\left[\mathrm{Fe}^{3+}\right]:\left[\mathrm{Cu}^{2+}\right]} \\
\text { added }(\mathrm{g} / \mathrm{l})\end{array}$ & $\begin{array}{l}{\left[\mathrm{H}_{2} \mathrm{SO}_{4}\right]} \\
\text { determined } \\
(\mathrm{g} / \mathrm{l})\end{array}$ & $\begin{array}{l}\text { Average }\left[\mathrm{H}_{2} \mathrm{SO}_{4}\right] \\
\text { determined }(\mathrm{g} / \mathrm{l})\end{array}$ & $\begin{array}{l}\% \\
\text { Difference }\end{array}$ & $\begin{array}{l}\text { R.S.D. for } \mathrm{H}_{2} \mathrm{SO}_{4} \\
\text { determinations } \\
(\%)\end{array}$ \\
\hline \multirow[t]{2}{*}{$* 5.34: 0: 0: 0$} & 5.34 & 5.34 & & \\
\hline & 5.34 & & & \\
\hline \multirow[t]{2}{*}{$5.34: 0: 0: 0$} & 5.14 & 5.19 & 2.81 & 1.36 \\
\hline & 5.24 & & & \\
\hline \multirow[t]{3}{*}{$5.34: 30: 30: 25$} & 5.62 & 5.62 & 5.24 & 1.51 \\
\hline & 5.53 & & & \\
\hline & 5.70 & & & \\
\hline \multirow[t]{4}{*}{$5.34: 0: 30: 0$} & 5.00 & 5.41 & 1.31 & 5.51 \\
\hline & 5.70 & & & \\
\hline & 5.40 & & & \\
\hline & 5.53 & & & \\
\hline
\end{tabular}

*true sulphuric acid value determined without potassium oxalate 


\subsubsection{Potassium Tartrate as Complexing Agent}

A GENCOR method used potassium tartrate for the determination of nickel in the presence of iron and it was thought the potassium tartrate could be used in the acid titrations as well. A saturated solution of potassium tartrate was used and the samples were titrated with sodium hydroxide.

The optimum potassium tartrate concentration was determined first (Table 4.5).

Table 4.5: Determination of the optimum concentration of potassium tartrate solution

\begin{tabular}{|l|l|l|}
\hline$\left[\mathrm{H}_{2} \mathrm{SO}_{4}\right]$ added $(\mathrm{g} / \mathbf{l})$ & $\begin{array}{l}\text { Potassium tartrate } \\
\text { concentration }(\mathrm{g} / \mathbf{l})\end{array}$ & $\begin{array}{l}{\left[\mathbf{H}_{2} \mathbf{S O}_{4}\right] \text { determined }} \\
(\mathrm{g} / \mathbf{l})\end{array}$ \\
\hline 5.23 & 300 & 5.46 \\
\hline 5.23 & 150 & 5.26 \\
\hline 5.23 & 75 & 5.23 \\
\hline 5.23 & 37.5 & 5.17 \\
\hline 5.23 & 0 & 5.23 \\
\hline
\end{tabular}

The optimum concentration of tartrate used was $75 \mathrm{~g} / \mathrm{l}$. The results were high and inaccurate (Table 4.6) when the acid is determined in the presence of interfering ions. Potassium tartrate was therefore not deemed an appropriate complexing agent. 
Table 4.6: Determination of sulphuric acid, in the presence of iron(III) and copper(II), using potassium tartrate

\begin{tabular}{|l|l|l|l||}
\hline \hline $\left.\mathrm{H}_{2} \mathrm{SO}_{4}\right]$ added $(\mathrm{g} / \mathrm{l})$ & {$[$ Ions] added $(\mathrm{g} / \mathrm{l})$} & $\begin{array}{l}{\left[\mathrm{H}_{2} \mathrm{SO}_{4}\right]} \\
\text { determined }(\mathrm{g} / \mathbf{l})\end{array}$ & \% Difference \\
\hline 5.23 & $30 \mathrm{~g} / \mathrm{l} \mathrm{Fe}$ & 7.97 & 52.39 \\
\hline 5.23 & $25 \mathrm{~g} / \mathrm{Cu}^{2+}$ & 6.37 & 21.80 \\
\hline 5.23 & $30 \mathrm{~g} / \mathrm{F} \mathrm{F}^{3+}, 25 \mathrm{~g} / 1 \mathrm{Cu}^{2+}$ & 8.98 & 71.70 \\
\hline 5.23 & none & 5.23 & 0 \\
\hline
\end{tabular}

\subsubsection{Failure of Complexometric Methods}

An examination of thermodynamic stabilities shows that iron(III) forms stable complexes with EDTA and oxalate $[38,39]$. Thermodynamic considerations alone would indicate that complexometric methods using EDTA or oxalate would mask the effect of the hydrolysable cations. The amount of base consumed in the titration should therefore be an indication of the amount of free acid in the sample. Varying results were found for the complexing of the cations with potassium oxalate. Since the hydrolysis reaction is the only source of protons it follows that the hydrolysis is not completely suppressed by the oxalate.

Thermodynamic stability constants can be transformed into 'conditional' stability constants $[38,39]$ that are more representative of the relative strengths of complexes 
formed by the masking reagents when the metal is in competition with the proton for the molecule of the complexing agent. Figure 4.1 illustrates the relationship between $\mathrm{pH}$ and conditional stability of selected metal-EDTA complexes.

Figure 4.1: Conditional stability constants, $K^{\prime}$, of selected metal-EDTA complexes as a function of $\mathrm{pH}[38]$

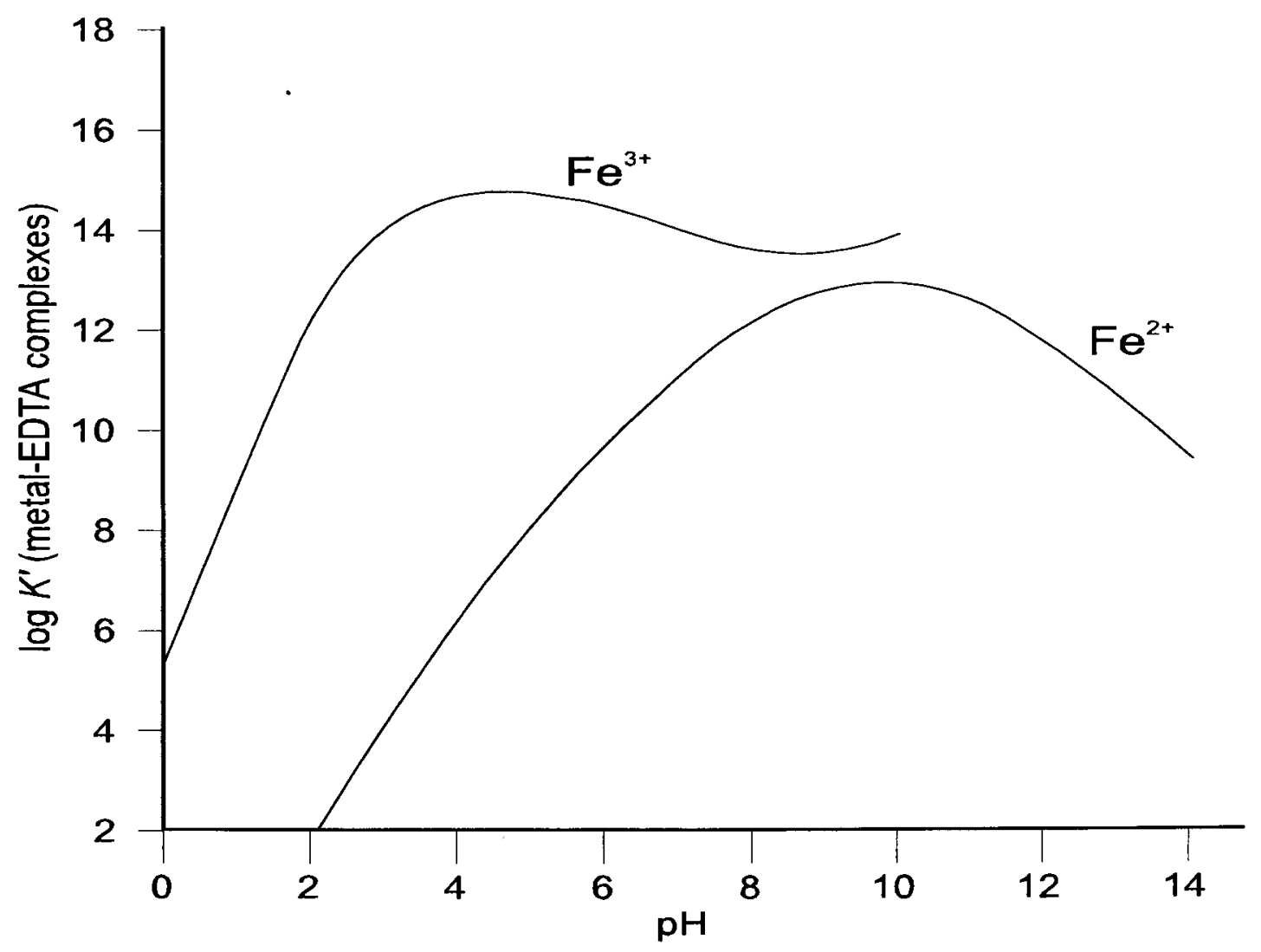

The stability of the main interferents reaches a local maximum at a $\mathrm{pH}$ value of 5 . Although methods that rely on the masking of hydrolysable cations are theoretically sound, they do not always work in practice. This may be due to the differences in 
concentrations and ionic strengths (and thus the activity coefficients of the species) of sample solutions. For the reaction with reactants $\mathrm{A}$ and $\mathrm{B}$ and products $\mathrm{C}$ and $\mathrm{D}$, the equilibrium constant, $\mathrm{K}_{\mathrm{eq}}$, is defined as:

$$
\mathrm{K}_{\mathrm{eq}}=\left(\mathrm{a}_{\mathrm{C}} \cdot \mathrm{a}_{\mathrm{D}}\right) /\left(\mathrm{a}_{\mathrm{A}} \cdot \mathrm{a}_{\mathrm{B}}\right)
$$

Where the activity of species $A$ is represented as $a_{A}$. The activity is defined as:

$\mathrm{a}_{\mathrm{A}}=[\mathrm{A}] \gamma_{\mathrm{A}}$

where $[\mathrm{A}]=$ concentration of species $\mathrm{A}$

$$
\gamma_{\mathrm{A}}=\text { activity coefficient of species } \mathrm{A}
$$

When the activities are expressed in terms of the concentrations of the species and the activity coefficients, the last equation can be rewritten.

$$
\mathrm{K}_{\mathrm{eq}}=([\mathrm{C}][\mathrm{D}] /[\mathrm{A}][\mathrm{B}]) \cdot\left(\gamma_{\mathrm{C}} \cdot \gamma_{\mathrm{D}}\right) /\left(\gamma_{\mathrm{A}} \cdot \gamma_{\mathrm{B}}\right)
$$

This is often abbreviated to

$$
\mathrm{K}_{\mathrm{eq}}=([\mathrm{C}][\mathrm{D}] /[\mathrm{A}][\mathrm{B}])
$$

on the assumption that the product of the activity coefficients is equal to unity. In concentrated solutions, the 'dilute solution' approximation fails because the product of the activity coefficients deviates from unity. Similarly, predictions from thermodynamic data may not directly apply to real solutions as not all factors are considered, which may 
explain the failure of masking methods, e.g. the potassium oxalate method, to completely remove the effect of high concentrations of hydrolysable cations.

Several other equilibria must be considered in complexometric masking reactions.

$$
\mathrm{MA}+\mathrm{H}_{2} \mathrm{O} \rightleftharpoons \mathrm{M}(\mathrm{OH})+\mathrm{H}^{+}+\mathrm{A}^{-}
$$

This equation represents one of the reactions involved in solution equilibria, and the set of equations given in Table 4.7 represents the most relevant solution equilibria [12].

Equation (1) excludes complexing of at least a fraction of the metal ions. In addition the effect of the other equilibria must also be taken into account. Species involved in these equilibria have a mutual dependence as they are common in more than one equation. At a $\mathrm{pH}$ value of 5.0, EDTA or oxalate will be partially protonated. At lower $\mathrm{pH}$ values, a greater fraction of the complexing agent will be protonated and will affect the effectiveness of the masking reagent. Above a $\mathrm{pH}$ value of 1 , hydrolysis becomes increasingly important. Conditions can be found such that the solution equilibria become negligible, and overall stoichiometry is represented by only the masking reaction, (Equation 1). Under these special conditions, the complexometric method may be used for the accurate estimation of the free acid concentration. Although the determination of free acids under special conditions has been proved, these methods are not generally valid. 
Table 4.7: Generalised solution equilibria for a special case for the monovalent, mononuclear metal cation $\left(\mathrm{M}^{+}\right)$and a monovalent anion, $\mathrm{A}^{-}$.

\begin{tabular}{|l|l||}
\hline EQUATION & NO. \\
\hline $\mathrm{M}^{+}+\mathrm{A}^{-} \leftrightarrow \mathrm{MA}$ & 1 \\
\hline $\mathrm{M}^{+}+\mathrm{HA} \leftrightarrow \mathrm{MHA}^{+}$ & 2 \\
\hline $\mathrm{M}^{+}+\mathrm{HA} \leftrightarrow \mathrm{H}^{+}+\mathrm{MA}$ & 3 \\
\hline $\mathrm{H}^{+}+\mathrm{A}^{-} \leftrightarrow \mathrm{HA}$ & 4 \\
\hline $\mathrm{H}^{+}+{\mathrm{MA} \leftrightarrow \mathrm{MHA}^{+}}$ & 5 \\
\hline $\left.\mathrm{M}^{+}+\mathrm{H}_{2} \mathrm{O} \leftrightarrow \mathrm{M}^{+} \mathrm{OH}\right)+\mathrm{H}^{+}$ & 6 \\
\hline $\mathrm{H}^{+}+\mathrm{OH}^{-} \leftrightarrow \mathrm{H}_{2} \mathrm{O}$ & 7 \\
\hline $\mathrm{H}_{2} \mathrm{SO}_{4} \leftrightarrow \mathrm{HSO}_{4}^{-}+\mathrm{H}^{+}$ & 8 \\
\hline $\mathrm{HSO}_{4}^{-} \leftrightarrow \mathrm{SO}_{4}^{2-}+\mathrm{H}^{+}$ & 9 \\
\hline \hline
\end{tabular}




\subsubsection{Conclusion}

Potassium oxalate and tartrate were investigated as complexing agents to aid in the masking of the hydrolysable cations. The potassium oxalate method gave varying results for the acid determination than expected. Potassium tartrate yielded results that were high and inaccurate for the acid determination in the presence of hydrolysable cations.

Thermodynamic considerations alone would indicate that complexometric methods would mask the effect of the hydrolysable cations. In practise this does not work which may be due to the differences in concentrations and ionic strengths of the sample solutions and 'theoretical' solutions for which the theory is derived. 


\subsection{The Effects of Dilution on Acid Determination}

Dilution can also be employed in analytical chemistry to reduce the interferent to levels with less pronounced effect on the accuracy of results. In this investigation the effect of dilution was investigated to decrease the cation concentration thereby decreasing the effects of the interfering cations. It was anticipated that the results would be more accurate. The differences for results done by the same method on diluted sample and undiluted sample were compared. The results in Table 4.8 confirm that dilution decreases the cation interference in samples with lower sulphuric acid content. It did not however hold true for samples with higher acid content. The values for acid concentration for undiluted and diluted sample differed significantly (Table 4.8). It was also different to the results obtained with an auto titrator. Values in Table 4.8 suggest the auto titrator values were more accurate than those obtained using a manual titration to a visual endpoint. 
Table 4.8: Determination of the effects of dilutions on interferents

\begin{tabular}{|c|c|c|c|c|c|}
\hline $\begin{array}{l}\text { Theoretical } \\
{\left[\mathrm{H}_{2} \mathrm{SO}_{4}\right](\mathrm{g} / \mathrm{l})}\end{array}$ & $\begin{array}{l}{\left[\mathrm{H}_{2} \mathrm{SO}_{4}\right]} \\
\text { added } \\
(\mathrm{g} / \mathrm{l})\end{array}$ & $\begin{array}{l}{\left[\mathrm{Fe}^{2+}\right]} \\
\text { added }(\mathrm{g} / \mathrm{l})\end{array}$ & Dilution & $\begin{array}{l}\text { Determined } \\
{\left[\mathrm{H}_{2} \mathrm{SO}_{4}\right] \text { added }} \\
(\mathrm{g} / \mathrm{l})\end{array}$ & $\begin{array}{l}\% \\
\text { Difference }\end{array}$ \\
\hline \multirow[t]{5}{*}{80} & 25.0 & 30.0 & 12.5 & 79.69 & 15.56 \\
\hline & None & None & 12.5 & 68.96 & 2.68 \\
\hline & None & None & None & 70.86 & \\
\hline & None* & None & 12.5 & 70.85 & 0.07 \\
\hline & None* & None & None & 70.80 & \\
\hline \multirow[t]{5}{*}{20} & 25.0 & 30.0 & 12.5 & 17.50 & 4.98 \\
\hline & None & None & 12.5 & 16.09 & 3.48 \\
\hline & None & None & None & 16.67 & \\
\hline & None* & None & 12.5 & 16.71 & 0.42 \\
\hline & None* & None & None & 16.78 & \\
\hline \multirow[t]{5}{*}{10} & 25.0 & 30.0 & 12.5 & 7.66 & 0 \\
\hline & None & None & 12.5 & 7.66 & 5.32 \\
\hline & None & None & None & 8.09 & \\
\hline & None* & None & 12.5 & 8.20 & 0.97 \\
\hline & None* & None & None & 8.28 & \\
\hline
\end{tabular}

*indicates determinations carried out on an automatic titrator 


\subsection{Effect of Neutral Salts}

Critchfield and Johnson $[13,14]$ reported on the ability to titrate weak bases in aqueous solutions with high concentrations of neutral salts. Neutral salts they defined as those derived from strong monoprotic inorganic acids and strong mono- or divalent inorganic bases. They stated that the apparent $\mathrm{pH}$ of the solution decreased linearly with increasing concentration of a neutral salt. Salts with higher positive heat of solution produced a greater change in $\mathrm{pH}$.

In the titration of aniline with hydrochloric acid the inflections on the potentiometric curve becomes more defined. Also in the presence of an excess of hydrochloric acid the initial $\mathrm{pH}$ of the solution decreases as the salt concentration increases. The hydrochloric acid behaved as a stronger acid in an aqueous solution that contains a neutral salt. In view of this Kubota and Costanzo [15] suggested the use of the same principles for the determination of free acid in the presence of hydrolysable cations. The idea was to use the increase in apparent acid activity to resolve the respective endpoints of the acid and the hydrolysable cations. They determined the free acid concentration in the presence of hydrolysable cations in solution with high concentration of lithium chloride. The apparent change in $\mathrm{pH}$ improves the shape of the inflections in the potentiometric curves, allowing a better distinction between the inflection of the free acid and that of small concentrations of metallic ions. They based their findings on the hydrated proton that was divested of part of its waters of hydration and consequently acquired greater activity or effective concentration. 
Lithium chloride is quite soluble in water up to a concentration of $16.5 \mathrm{~mol} / \mathrm{l}$ at ambient temperature. Hogfelt and Leifer [16] showed that the average hydration of lithium drops from 6 water units per lithium ion in dilute solutions, to 3.1 in concentrated solutions, while the chloride hydration drops from 2 to 0 in the same range. Above $6 \mathrm{~mol} / \mathrm{l}$ lithium chloride, lithium ions do not have the maximum hydration and would be expected to compete with protons for any available water molecules, and more so with hydrolysable cations which do not retain waters of hydration as readily as lithium in solution. This changes the activity of these cations. This theory is strengthened by the observation that, on adding lithium chloride to a iron(III) solution, the yellow colour, which is due to the iron(III) chloro complex, deepened visibly. Also addition of lithium chloride to a pink cobalt(II) sulphate solution caused the solution to turn an intense blue colour, due to the formation of the cobalt(II) chloro complex and the consequent displacement of water.

\subsubsection{Lithium Chloride Titrations}

The use of high concentrations of lithium salt in acid titrations with high concentrations of hydrolysable cations was tried. A method based on this theory suggested that the volume of saturated lithium chloride solution added must be three times the volume of sample added. It was found however that the optimum volume of lithium chloride solution was five times the sample volume. Smaller volumes (in the presence of iron(III)) yielded results that were low and bigger volumes (in the presence of iron(III)) did not improve the results significantly (Table 4.9). In addition $50 \mathrm{ml}$ was a large enough volume for the electrode to be immersed in liquid. The final solution volume was not 
diluted since this affected the accuracy of the results as the lithium chloride concentration decreases.

Table 4.9: Determination of optimum Lithium Chloride volume

\begin{tabular}{||l|l|l|l||}
\hline$\left[\mathrm{H}_{2} \mathrm{SO}_{4}\right]$ added $(\mathrm{g} / \mathbf{l})$ & {$\left[\mathrm{Fe}^{3+}\right]$ added $(\mathrm{g} / \mathbf{l})$} & $\begin{array}{l}\text { Volume of Lithium } \\
\text { Chloride added }\end{array}$ & $\begin{array}{l}{\left[\mathbf{H}_{2} \mathrm{SO}_{4}\right]} \\
\text { determined }(\mathrm{g} / \mathrm{l})\end{array}$ \\
\hline 5.2 & 0 & 0 & 5.22 \\
\hline 5.2 & 0 & 10 & 5.21 \\
\hline 5.2 & 0 & 50 & 5.20 \\
\hline 5.2 & 0 & 60 & 5.22 \\
\hline 5.2 & 30 & 10 & 4.77 \\
\hline 5.2 & 30 & 50 & 5.21 \\
\hline 5.2 & 30 & 60 & 5.20 \\
\hline
\end{tabular}

The original method also recommended that the $\mathrm{pH}$ of the $13 \mathrm{~mol} / 1$ lithium chloride solution be adjusted to the $\mathrm{pH}$ of neutralisation $(\mathrm{pH}=4)$. This leads to unnecessary dilution of the sample. The lithium chloride solution used for the purposes of this investigation was $13 \mathrm{~mol} / \mathrm{l}$ but the $\mathrm{pH}$ had not been adjusted.

The percentage difference between the average CP. grade and average A.R. grade values with the average A.R. grade value taken as the true value is $0.20 \%$. The relative standard deviation for the two grades of reagent is $0.6 \%$. This indicates that the accuracy and 
precision of the results were not affected when A.R. and CP. grades lithium chloride was used as indicated in Table 4.10. In addition results obtained using a $0.1 \mathrm{~mol} / \mathrm{l}$ sodium hydroxide titrant were not significantly different from that obtained using a $0.1 \mathrm{~mol} / \mathrm{l}$ sodium hydroxide $/ 10 \mathrm{~mol} / \mathrm{l}$ lithium chloride titrant.

Table 4.10: Determination of sulphuric acid using CP. and A.R. grade lithium chloride

\begin{tabular}{|l|l|l|l||}
\hline$\left[\mathrm{H}_{2} \mathrm{SO}_{4}\right]$ & Accuracy and & {$\left[\mathrm{H}_{2} \mathrm{SO}_{4}\right]$} & Precision of the \\
determined using & precision of the & determined using \\
AR LiCl & results & $\begin{array}{l}\text { C.P. grade LiCl } \\
(\mathrm{g} / \mathrm{l})\end{array}$ & \\
$(\mathrm{g} / \mathrm{l})$ & & 5.01 & \\
\hline 5.00 & R.S.D. $=0.62 \%$ & 5.07 & R.S.D. $=0.65 \%$ \\
\hline 5.03 & & 5.08 & \\
\hline 5.08 & & 5.03 & \\
\hline 5.04 & & & \\
\hline 5.01 & & & \\
\hline
\end{tabular}

Titration of the various cation solutions with lithium chloride yielded satisfactory results. Even the manganese(II) and iron(III), the two cations which cause the most serious interference in the determinations gave reasonably precise results(Table 4.11).

Solomons [4] found that solutions containing iron(II) should be stored under anaerobic conditions to prevent oxidation of the iron(II) to iron(III), and avoid low results. This 
investigation showed that if concentrated solutions of lithium chloride are used, the analysis is not significantly affected by the oxidation of the ferrous ion. Free acid titrations in the presence of hydrolysable cations could be successfully performed in the presence of lithium chloride solution.

Table 4.11: Sulphuric acid determination in the presence of various cations using lithium chloride.

\begin{tabular}{||l|l|l||}
\hline Solution and [cation] (g/l) & {$\left[\mathrm{H}_{2} \mathrm{SO}_{4}\right]$ determined (g/l) } & \% Difference \\
\hline $\mathrm{H}_{2} \mathrm{SO}_{4}$ & 4.81 (determined in water) & \\
\hline $\mathrm{H}_{2} \mathrm{SO}_{4}$ & 4.81 (determined in lithium chloride) & \\
\hline $\mathrm{NiSO}_{4}(98)$ & 4.69 & 2.5 \\
\hline $\mathrm{Ni}\left(\mathrm{NO}_{3}\right)_{2}(386)$ & 4.69 & 2.5 \\
\hline $\mathrm{CoSO}_{4}(102)$ & 4.69 & 2.5 \\
\hline $\mathrm{CoCl}_{2}(152)$ & 4.69 & 2.5 \\
\hline $\mathrm{MnCl}_{2}(198)$ & 4.69 & 2.5 \\
\hline${\mathrm{Mn}\left(\mathrm{NO}_{3}\right)_{2}(746)}$ & 5.05 & 5.0 \\
\hline $\mathrm{CuSO}_{4}(53)$ & 4.51 & 6.2 \\
\hline $\mathrm{Fe}_{\left(\mathrm{NO}_{3}\right)_{3}(90.4)}$ & 5.20 & 8.1 \\
\hline $\mathrm{FeSO}_{4}(30.4)$ & 4.76 & 1.0 \\
\hline
\end{tabular}




\subsubsection{Evaluation of Other Neutral salts}

Acid solutions containing high concentrations of transition metals were titrated in the presence of high concentrations of neutral salts.

The addition of high concentration of a neutral salt may work for two reasons: (1) the high concentration of the chloride ion might prevent hydrolysis of the cation through the formation of stable chloro-complexes, or (2) there is competition for water molecules for hydrolysis where the concentration of the cation is the determining factor.

To determine which factor is dominant, various highly soluble salts of chloride were tested. If only the chloride ion concentration is important, lithium, zinc, and magnesium chloride would give comparative results due to their high solubilities. The acid concentrations obtained with the different salts were compared with those obtained using lithium chloride (Table 4.12). 
Table 4.12: Results of free acid titration in the presence of neutral salts

\begin{tabular}{||l|l|l||}
\hline Neutral Salt & $\begin{array}{l}\mathbf{H}_{2} \mathrm{SO}_{4} \text { Solution } \\
\mathbf{( 0 . 0 4 8} \mathrm{mol} / \mathbf{l}) \\
\text { No cations present }\end{array}$ & $\begin{array}{l}\text { Sample GF830/6 } \\
\mathbf{( 0 . 1 0 0 M )} \\
\text { Iron(III) present }\end{array}$ \\
\hline $\mathrm{LiCl}$ & $0.048 \mathrm{~mol} / \mathrm{l}$ & $0.100 \mathrm{~mol} / 1$ \\
\hline $\mathrm{NaCl}$ & $0.026 \mathrm{~mol} / 1$ & $0.135 \mathrm{~mol} / 1$ \\
\hline $\mathrm{CaCl}_{2}$ & $0.052 \mathrm{~mol} / 1$ & $0.149 \mathrm{~mol} / \mathrm{l}$ \\
\hline $\mathrm{MgCl}_{2}$ & $0.049 \mathrm{~mol} / \mathrm{l}$ & $0.121 \mathrm{~mol} / 1$ \\
\hline $\mathrm{ZnCl}_{2}$ & No endpoint & No endpoint \\
\hline
\end{tabular}

The routine application of lithium chloride is restricted by its high cost. The volume of samples analysed in our laboratories for free acid warranted the investigation into more cost-effective alternatives.

The calcium chloride reagent was not suitable due to the precipitation of calcium sulphate in the presence of sulphuric acid. Zinc and calcium chloride resulted in poor titration curves (Figure 4.2). The sodium chloride curve gave little useful information. Only magnesium chloride gave results comparable to those obtained for lithium chloride, from this it was concluded that the concentration of the chloride ion was not the dominant effect in the titration. The hydrolysis effect of the cation was considered next by a qualitative comparison of the titration curves (Figure 4.2). From the graphs it was evident that concentrated solutions of magnesium and lithium chloride resulted in a more 
pronounced resolution of the inflection during the titration of free acid, even when the concentration of hydrolysable cations was high. The curves for zinc and sodium chloride gave little useful information.

To investigate the effect of chloride different lithium salts were used in the titration of a synthetic solution. The results were compared to those obtained using lithium chloride. The salts chosen were: lithium nitrate and lithium sulphate. Close inspection of the titration curves (Figure 4.3) reveals that the starting $\mathrm{pH}$ 's for the different test solutions were different. The difference can be correlated to the difference in the heat of the salts. Critchfield and Johnson $[13,14]$ found that the higher the heat of solution of a neutral salt the greater the decrease in $\mathrm{pH}$ of the sample solution. Lithium chloride having the highest heat of solution has the lowest starting $\mathrm{pH}$ and result in a curve which has a neutralisation incline that is more pronounced and occurs over a broader $\mathrm{pH}$ band than that of lithium nitrate and sulphate. All three lithium salts gave acceptable results.

The situation was different when the same lithium salts were tested on a synthetic solution containing $11 \mathrm{~g} / 1$ iron(III). Lithium chloride gave an acceptable result. The lithium sulphate and nitrate gave no endpoints whatsoever, all that is seen on the titration curves is the steady increase of the $\mathrm{pH}$ of the sample solution with increase in titrant volume. This observation can be explained by the difference in starting $\mathrm{pH}$ for each of the salts. Since the starting $\mathrm{pH}$ of the lithium sulphate and nitrate is higher the acid endpoint is merged into the cation hydrolysis endpoint and hence no clear acid endpoint is seen. In the case of the lithium chloride the lower starting $\mathrm{pH}$ means that the curve is more 
resolved and the acid endpoint can be seen before the cation hydrolysis occurs. This could be due to the bond formed between the chloride ion and the interfering cation which influences hydrolysis. The lithium ion aids in the dehydration of the interfering cation and the chloride ion forms a complex that keeps the interfering cation in solution for longer, thereby allowing the acid endpoint to be seen clearly without any interference form the cation hydrolysis. Whatever the reason, it is clear that both the lithium ion and the chloride ion are important in the mechanism that allows the titrations in the presence of hydrolysable cations. The effect of the two ions are not mutually exclusive.

The effects seen for the various neutral salts could be explained by the activity of the proton being enhanced by the competing hydrolysis postulate. This is supported by other physical constants such as the value of the heat of solution of the cations (a measure of the enthalpy released when the salt dissolves in water) and on an even more fundamental level, by considering the charge and volume of the respective cations. 
Figure 4.2: Comparison of different titration curves in the presence of different neutral salts

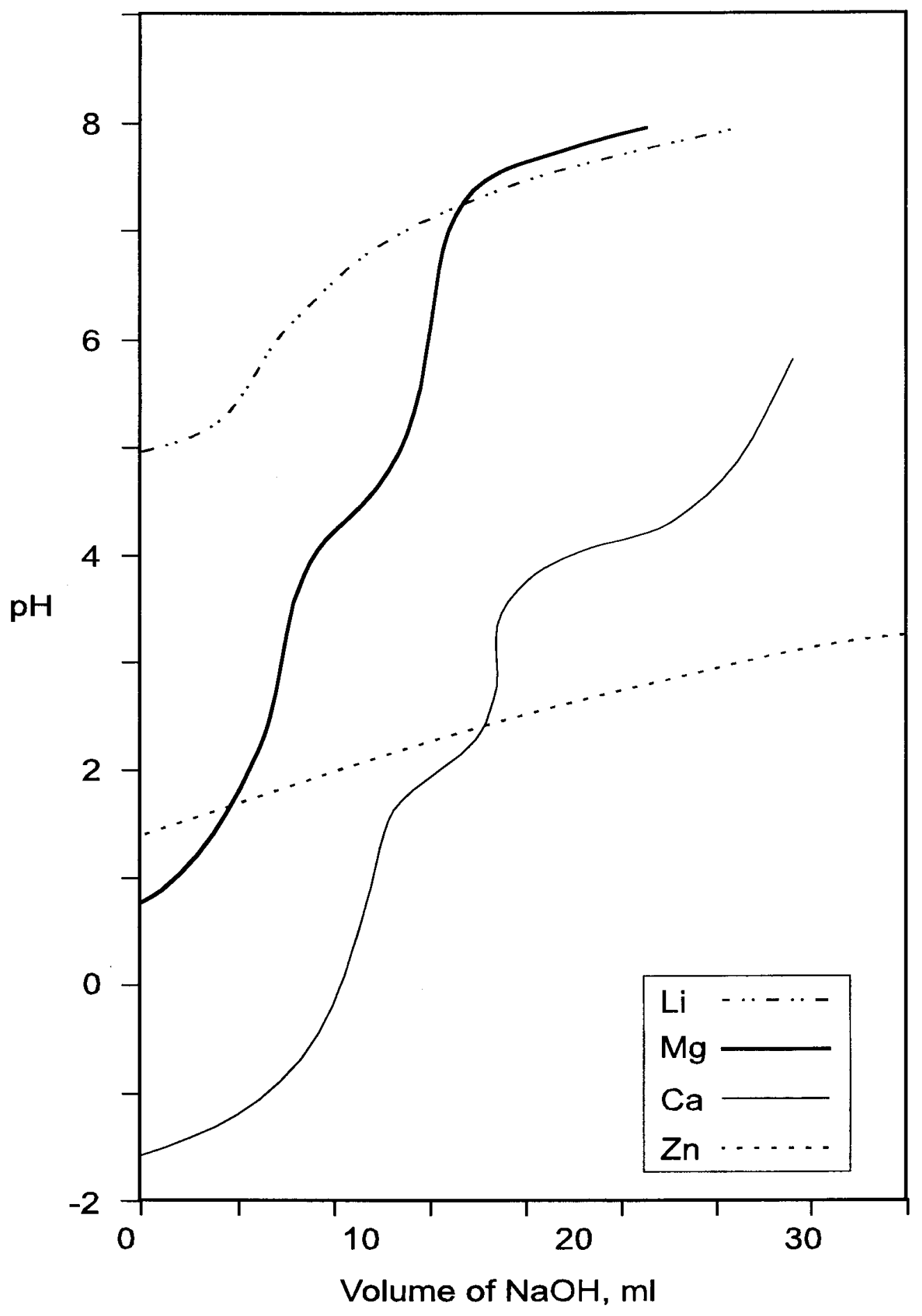


Figure 4.3: Curves for acid determination using different lithium salts

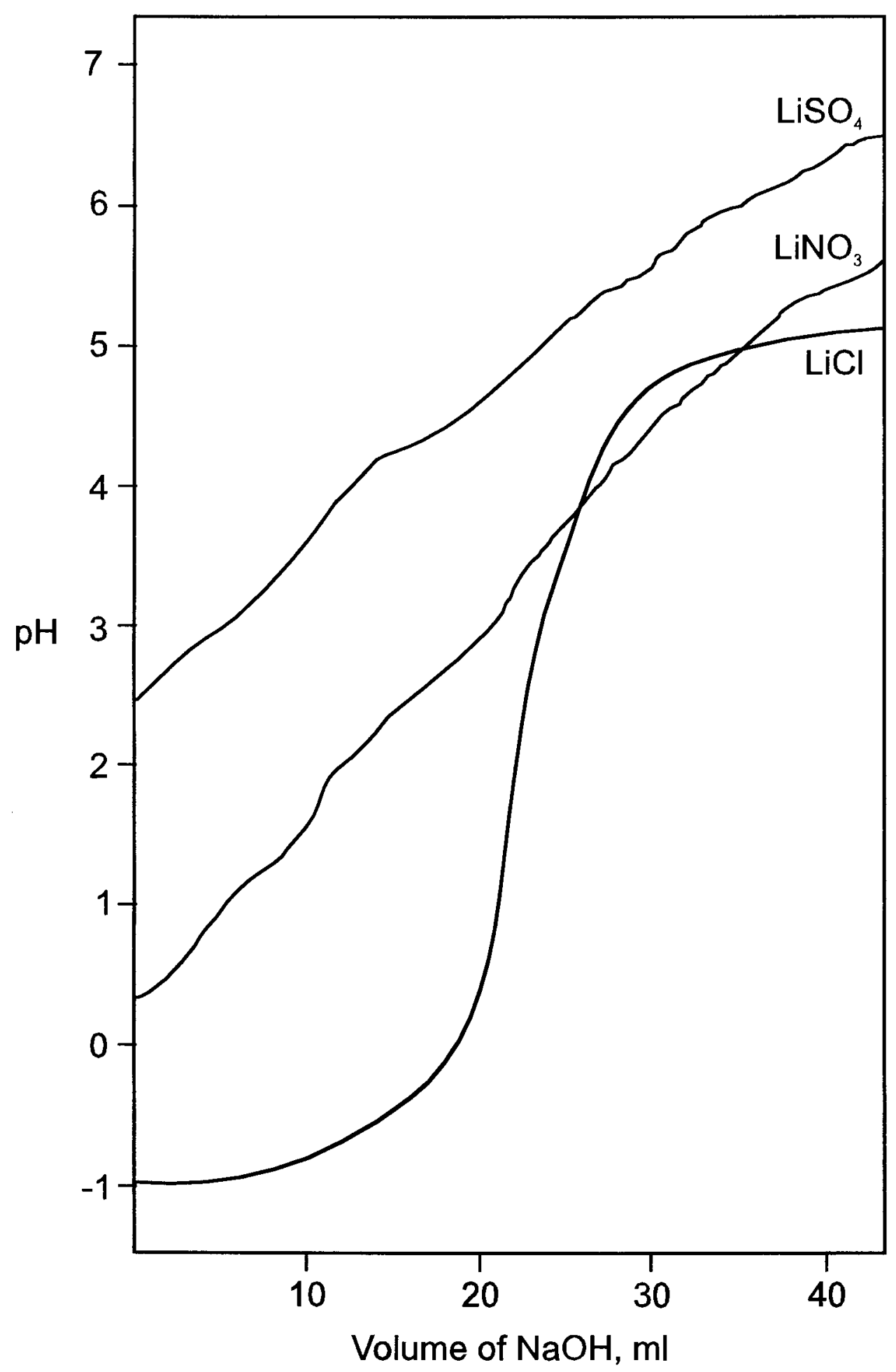


The heat of solution depends on the temperature of the solution, the concentration of the salt, the availability of water, and various other factors. This release of energy is an indication of the extent of hydrolysation involved and thus of the hydration of the cation. Table 4.13 displays the solubility of various neutral salts as well as the respective heats of solution.

Table 4.13: Solubility and heat of solution of some chloride salts [22]

\begin{tabular}{|l|l|l||}
\hline Neutral Salt & Solubility at $\mathbf{2 0}^{\circ} \mathbf{C}$, in $\mathbf{~ m o l ~} \mathbf{C l}$ & Heat of solution in $\mathbf{1 0}^{-3} \mathbf{~} \mathbf{J} / \mathbf{m o l}$ \\
\hline $\mathrm{LiCl}$ & 16.5 & 35.6 \\
\hline $\mathrm{NaCl}$ & 6.1 & -5.3 \\
\hline $\mathrm{MgCl}_{2}$ & 18 & 150.6 \\
\hline $\mathrm{CaCl}_{2}$ & 25.5 & 75.3 \\
\hline $\mathrm{ZnCl}_{2}$ & 63.4 & 65.8 \\
\hline
\end{tabular}

Table 4.14 displays the charge and relative size of the cations under consideration. In the hydration of the cation, the charge to volume is quite important. A small, highly charged cation (hard cation) will have a greater affinity for the water molecules than a large, singly charged cation (soft cation). Salts with the highest positive heat of solution and with small cation radii have greater affinity for water, and would tend to compete with both the hydrated proton and other cations for water molecules. The proton being harder would enter the neutralisation process first. 
Table 4.14: Cationic radii of some elements [27]

\begin{tabular}{||l|l|l||}
\hline \hline Cation & Charge & Size in pm (four co-ordination) \\
\hline $\mathrm{Li}$ & +1 & 73 \\
\hline $\mathrm{Ka}$ & +1 & 113 \\
\hline $\mathrm{NH}_{4}$ & +1 & 151 \\
\hline $\mathrm{Mg}$ & +1 & 130 \\
\hline $\mathrm{Ca}$ & +2 & 71 \\
\hline $\mathrm{Fe}$ & +2 & 114 \\
\hline $\mathrm{Cu}$ & +2 & 77 \\
\hline $\mathrm{Co}$ & +2 & 71 \\
\hline $\mathrm{Zn}$ & +2 & 72 \\
\hline $\mathrm{Al}$ & +2 & 74 \\
\hline $\mathrm{Fe}$ & +3 & 53 \\
\hline \hline
\end{tabular}

The combination of the values in Tables 4.13 and 4.14 suggests that magnesium, aluminium, or iron could be considered as substitutes for lithium. Aluminium and iron were not chosen for further investigation. The small radius of the iron(III) and aluminium cations, and their large charge, makes them hard cations and they are able to compete with the protons for water molecules and hence they are hydrolysed. Hydrolysation of these ions produces protons which results in high titres during the titration of free acid. Also aluminium has many side reactions in water and therefore was not considered in this investigation. 
Initial tests showed that magnesium chloride gave comparable results to lithium chloride when tested on synthetic and sample solutions containing cations. Also the heat of solution, charge and volume all indicate that magnesium chloride could be a substitute for lithium chloride. Magnesium chloride was therefore chosen for further tests in the free acid determination. It resulted in titration curves with more pronounced inflection points than lithium. Lithium chloride was therefore replaced with magnesium chloride in the free acid determination.

Magnesium nitrate was also tested to see if the same effect as that obtained with lithium and it's salts was observed. Titrations using magnesium nitrate don't show any endpoints whereas the same titration with magnesium chloride gives clear endpoints. It was once again evident that the cations form a strong bond with the chloride anion that keeps them in solution long enough for the acid endpoint to be determined.

Magnesium chloride is available in anhydrous and hexahydrated forms, both of which are deliquescent and form saturated solutions if left standing in a moist atmosphere. Although it would have been preferable to use the anhydrous form as the dehydrating agent during free acid titration, it is expensive and has low solubility in water. The hexahydrated form was therefore used throughout this project. 


\subsubsection{Conclusion}

Lithium chloride was investigated to determine the free acid concentration in the presence of hydrolysable cations. Lithium chloride changes the apparent $\mathrm{pH}$ of a solution and improves the shape of the inflections in the potentiometric curves, allowing a better distinction between the inflection of the free acid and that of the hydrolysable cations. The conditions for the use of lithium chloride was determined. The optimum volume of lithium chloride to be used was found to be $50 \mathrm{ml}$ of the saturated solution. It was found to be unnecessary to $\mathrm{pH}$ the lithium chloride solution to that of the endpoint. Also the use of $0.1 \mathrm{~mol} / \mathrm{l}$ sodium hydroxide titrant gave the same results as that obtained using 0.1 mol/l sodium hydroxide/ $10 \mathrm{~mol} / 1$ lithium chloride titrant. The use of $\mathrm{CP}$. grade lithium chloride gave the same results as A.R grade lithium chloride. When lithium chloride was used the free acid analysis in the presence of various cations gave satisfactory results.

The cost of lithium chloride inhibits its routine application and therefore a cost-effective alternative had to be found. It would seem that the lithium ion aids in the dehydration of the interfering cation and the chloride ion forms a complex with the interfering cation thereby keeping it in solution longer. The replacement neutral salt would have to satisfy both these criteria in order to be successful.

Factors such as heat of solution, solubility, and charge to size ratio indicate that iron, magnesium and aluminium could be considered as substitutes for lithium. Iron and aluminium are hydrolysed in the free acid determination, hence magnesium chloride was 
chosen for further investigation. Magnesium chloride gave comparable results to lithium chloride and was therefore chosen as the neutral salt for free acid determination. 


\subsection{Non-Aqueous Titrations}

The problems experienced during the titration of free acid in the presence of hydrolysable cations is clearly influenced by the hydration of certain cations. In the presence of water, hydrated cations act as a source of protons (equation 4.8 ) leading to erroneous titration results.

$\mathrm{M}\left(\mathrm{H}_{2} \mathrm{O}\right)_{\mathrm{n}}{ }^{\mathrm{z}^{+}} \rightleftharpoons \quad \mathrm{M}\left(\mathrm{H}_{2} \mathrm{O}\right)_{\mathrm{n}-1}(\mathrm{OH})^{(\mathrm{z}-1)}+\mathrm{H}^{+}{ }_{(\mathrm{aq})}$

It was thought that in the absence of water, the cations are not hydrated and, with the protons, are exposed to neutralisation. An indication of a solvents ability to solvate ionic species is given by the dielectric constant of the solvent [34]. The higher the dielectric constant the more reactive the respective ions. Therefore the behaviour of the cations and the protons in different solvents should theoretically have the same effect as large concentrations of neutral salts, and should inhibit the hydration of the hydrolysable cations.

The acidity and basicity of the sample can be enhanced by using a suitable non-aqueous medium [28]. Non-aqueous titrations are well known in the literature [28,41-44]. Titration of weak acids or bases can be difficult since only a small or no inflection is obtained in a titration curve. 
The Lowry- Bronsted concept explains this observation:

$$
\text { Acid } \rightleftharpoons \quad \text { base }+\mathrm{H}^{+}
$$

From this it is apparent that the base conjugate to a strong acid will be weak. The effect of the solvent on the equilibrium will be governed by its own proton accepting or donating properties, such that the strength of any acid or base is related to the solvent employed. The consequence of this is that acids and bases which are weak in water, and hence difficult to titrate, may be enhanced in strength by a suitable choice of solvent.

\subsubsection{Cyclohexylamine}

The titration of sulphuric acid and nitric acid with cyclohexylamine in a non-aqueous environment is described in the literature [41]. Due to the diprotic nature of sulphuric acid, two equivalent points were observed.

The first endpoint indicates the dissociation of the first proton. The difference between the first and second endpoints corresponds to the remaining proton of sulphuric acid. In the calculation of the result, the first equivalent point can be doubled or the second incline can indicate the endpoint. The titration of sulphuric acid leach samples containing high concentrations of iron(III), led to very poor reproducibility as the first equivalence point was almost always obscured by the presence of iron(III). 
Figure 4.4 shows a comparison between the cyclohexylamine in methanol (non-aqueous titration) of a sample and the respective sodium hydroxide in water (aqueous) titration. Although it was clear that the non-aqueous curve shows potential the inflections were not as well defined as in the aqueous titrations.

Figure 4.4: Non-aqueous (cyclohexylamine in methanol) versus non-aqueous titration (sodium hydroxide in water)

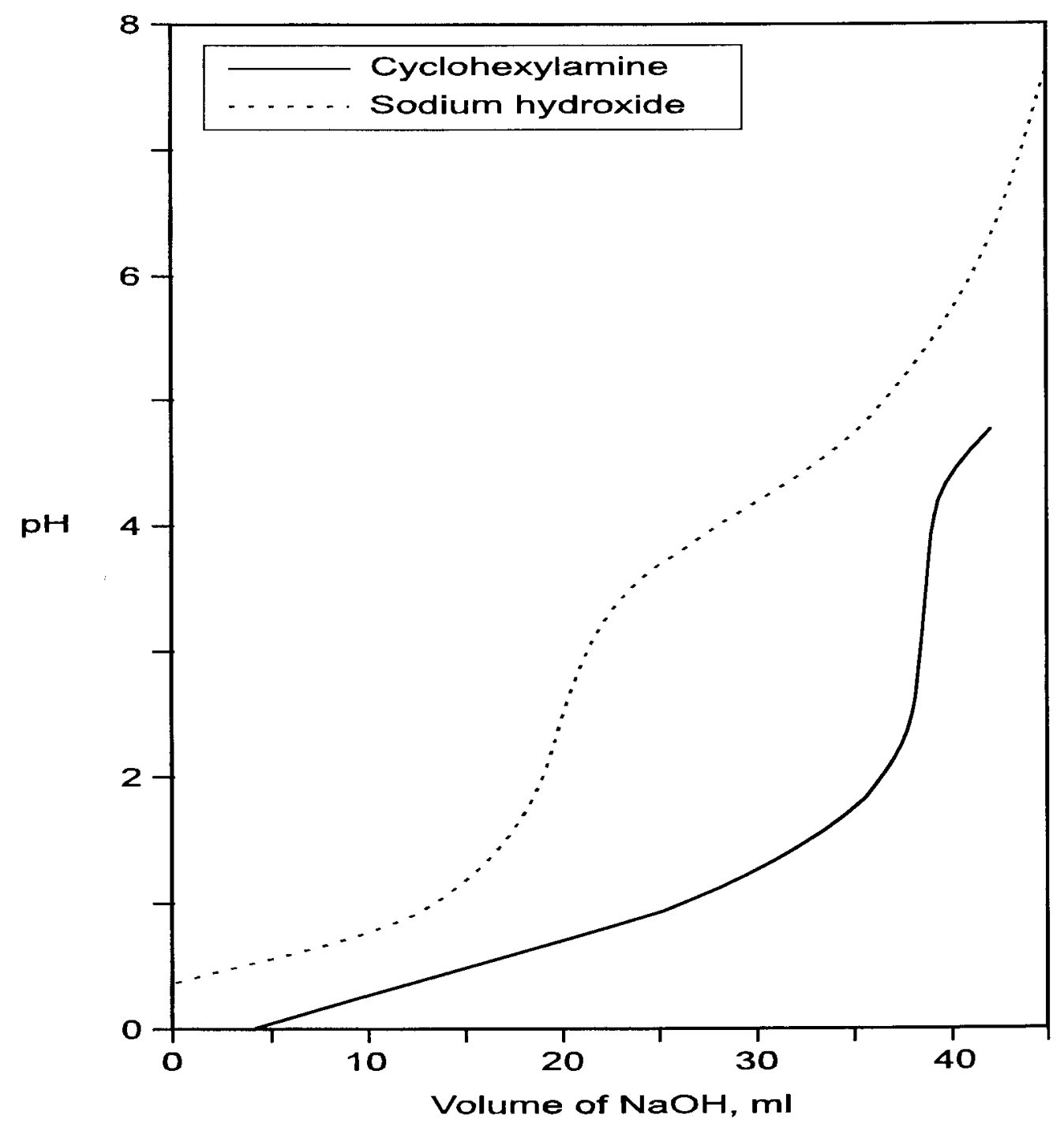




\subsubsection{Methanol}

Different solutions containing nickel(II), cobalt(II), manganese(II), copper(II), iron(III), and iron(II) were titrated with methanol. It was thought that the methanol would improve the equivalence points due to its dielectric constant of $32.6[28,42,45]$.

The curves are not very clearly defined. The results are satisfactory for the acid, nickel and cobalt solutions only (Table 4.15). Figure 4.5 shows a comparison of the nonaqueous methanol titrations for nickel (titration where an endpoint was seen) and copper (titration where no endpoint was not seen) and the corresponding aqueous titration. 
Table 4.15: Acid titration using methanol

\begin{tabular}{||l|l|l||}
\hline \hline Solution & {$\left[\mathbf{H}_{2} \mathrm{SO}_{4}\right]$ determined $(\mathbf{g} / \mathbf{l})$} & \% Difference \\
\hline $\mathrm{H}_{2} \mathrm{SO}_{4}$ (water as solvent) & 4.81 & \\
\hline $\mathrm{H}_{2} \mathrm{SO}_{4}$ & 4.69 & 2.5 \\
\hline $\mathrm{H}_{2} \mathrm{SO}_{4}$ & 4.69 & 2.5 \\
\hline $\mathrm{H}_{2} \mathrm{SO}_{4}$ & 4.72 & 1.8 \\
\hline $\mathrm{Ni}\left(\mathrm{NO}_{3}\right)_{2}$ & 5.05 & 5.0 \\
\hline $\mathrm{Co}\left(\mathrm{NO}_{3}\right)_{2}$ & 4.69 & 2.5 \\
\hline $\mathrm{Mn}\left(\mathrm{NO}_{3}\right)_{2}$ & 5.29 & 10.0 \\
\hline $\mathrm{CuSO}$ & & \\
\hline $\mathrm{Fe}\left(\mathrm{NO}_{3}\right)_{3}$ & Inflections not clear & \\
\hline $\mathrm{FeSO}$ & & Inflections not clear \\
\hline
\end{tabular}


Figure 4.5: Non-aqueous titrations for nickel(II) and copper(II)

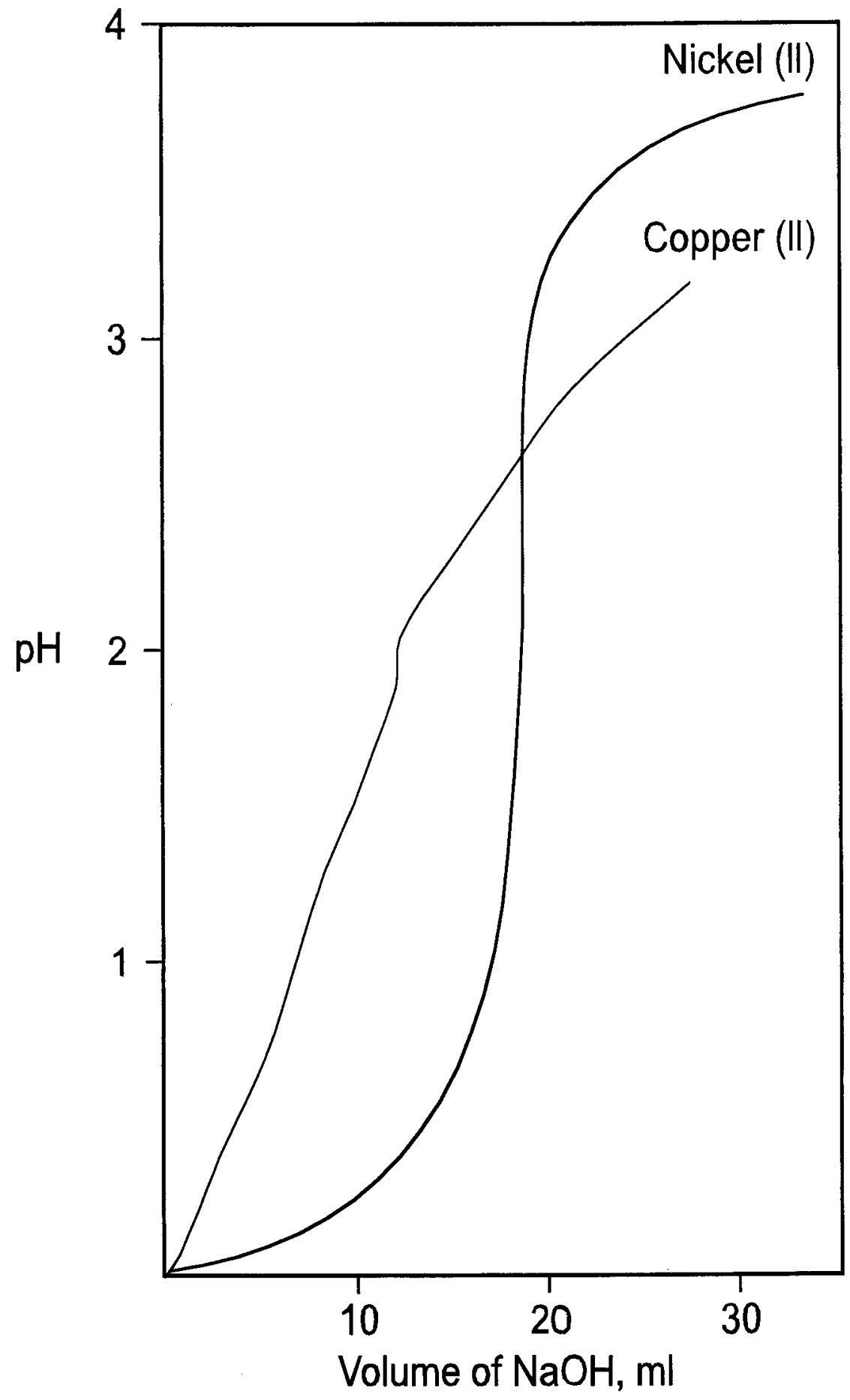




\subsubsection{Pyridine}

Pyridine has a sufficiently high dielectric constant (12.3) [45] to yield stable potentiometric readings. It could also therefore be used to enhance the titration of strong, medium and weak acids [42].

The same cation solutions were titrated with pyridine. The endpoints were not clearly defined in that there were no steep inclines. For the acid solution alone the result is comparable to that obtained with acid and water but the percentage difference is higher than that usually obtained (Table 4.16).

Table 4.16: Acid titrations using pyridine.

\begin{tabular}{||l|l|l||}
\hline Solution & {$\left[\mathrm{H}_{2} \mathrm{SO}_{4}\right]$ determined (g/l) } & \% Difference \\
\hline $\mathrm{H}_{2} \mathrm{SO}_{4}$ (water as solvent) & 4.81 & \\
\hline $\mathrm{H}_{2} \mathrm{SO}_{4}$ & 5.04 & 4.8 \\
\hline $\mathrm{Ni}\left(\mathrm{NO}_{3}\right)_{2}$ & Inflections not clear & \\
\hline $\mathrm{Co}\left(\mathrm{NO}_{3}\right)_{2}$ & Inflections not clear & \\
\hline $\mathrm{Mn}\left(\mathrm{NO}_{3}\right)_{2}$ & Inflections not clear & \\
\hline $\mathrm{CuSO}$ & & \\
\hline $\mathrm{Fe}\left(\mathrm{NO}_{3}\right)_{3}$ & Inflections not clear & \\
\hline $\mathrm{FeSO}$ & Inflections not clear & \\
\hline
\end{tabular}




\subsubsection{Acetone}

A number of acids have been titrated with success in acetone due to its high polarisability (dielectric constant $=20.7)[42,45]$. It was thought that due to its polarisability acetone should inhibit cation hydrolysation.

Titrations using acetone did not work for the cation solutions manganese(II), copper(II), iron(III), iron(II) since they were all precipitated when the acetone was added to the solutions. The nickel(II), cobalt(II) and straight acid solutions yielded acceptable results as indicated by Table 4.17. 
Table 4.17: Acid determination with acetone

\begin{tabular}{|c|c|c|}
\hline Solution & {$\left[\mathrm{H}_{2} \mathrm{SO}_{4}\right]$ determined $(\mathrm{g} / \mathrm{l})$} & $\%$ Difference \\
\hline $\begin{array}{l}\mathrm{H}_{2} \mathrm{SO}_{4} \text { (determined with } \\
\text { water as solvent) }\end{array}$ & 4.81 & \\
\hline $\mathrm{H}_{2} \mathrm{SO}_{4}$ & 4.81 & 0 \\
\hline $\mathrm{Ni}\left(\mathrm{NO}_{3}\right)_{2}$ & 4.81 & 0 \\
\hline $\mathrm{Co}\left(\mathrm{NO}_{3}\right)_{2}$ & 4.61 & 4.2 \\
\hline $\mathrm{Mn}\left(\mathrm{NO}_{3}\right)_{2}$ & 5.28 & 8.9 \\
\hline $\mathrm{CuSO}_{4}$ & Inflections not clear & \\
\hline $\mathrm{Fe}\left(\mathrm{NO}_{3}\right)_{3}$ & Inflections not clear & \\
\hline $\mathrm{FeSO}_{4}$ & Inflections not clear & \\
\hline
\end{tabular}

\subsection{5 t-Butyl Alcohol}

$t$-butyl alcohol is less acidic than the lower primary and secondary alcohols, probably because of the inductive effect of the 3 methyl groups on the carbon atom to which the hydroxyl group is attached. Therefore very weak acids can be titrated in t-butyl alcohol with good results. It was suggested [42] that sulphuric acid may be quantitatively titrated in t-butyl alcohol.

The titration results indicate that for the sulphuric acid, nickel(II), and manganese(II) solutions the results are far too high for quality purposes (Table 4.18). A percentage 
difference of less than $10 \%$ is considered acceptable. Copper(II), iron(III), and iron(II) gave curves with no clear inclines.

Table 4.18: Acid determination with $t$-butyl alcohol

\begin{tabular}{|c|c|c|}
\hline Solution & ( $\left[\mathrm{H}_{2} \mathrm{SO}_{4}\right]$ determined $(\mathrm{g} / \mathrm{l})$ & \% Difference \\
\hline $\begin{array}{l}\mathrm{H}_{2} \mathrm{SO}_{4} \text { (determined with } \\
\text { water as solvent) }\end{array}$ & 4.81 & \\
\hline $\mathrm{H}_{2} \mathrm{SO}_{4}$ & 5.45 & 13.3 \\
\hline $\mathrm{Ni}\left(\mathrm{NO}_{3}\right)_{2}$ & 5.71 & 18.7 \\
\hline $\mathrm{Co}\left(\mathrm{NO}_{3}\right)_{2}$ & 5.07 & 5.4 \\
\hline $\mathrm{Mn}\left(\mathrm{NO}_{3}\right)_{2}$ & 5.91 & 22.9 \\
\hline $\mathrm{CuSO}_{4}$ & Inflections not clear & \\
\hline $\mathrm{Fe}\left(\mathrm{NO}_{3}\right)_{3}$ & Inflections not clear & \\
\hline $\mathrm{FeSO}_{4}$ & Inflections not clear & \\
\hline
\end{tabular}

\subsubsection{Conclusion}

The problems experienced during free acid titrations are influenced by cation hydration. It was thought that in the absence of water, the cations are not hydrated and, with the protons, are exposed to neutralisation. The dielectric constant indicates the solvents ability to solvate ionic species, and therefore the higher the dielectric constant the more reactive the respective ions. It was therefore thought that the behaviour of the cations and 
protons in various solvents should theoretically have the same effect as high concentrations of neutral salts, and should inhibit the hydration of the hydrolysable cations. For some of the cation solutions the titration curves were enhanced in the presence of the solvents. It is clear that non-aqueous titrations show potential in some instances, although the curves are not always as clearly defined as in aqueous titrations. From the organic solvents tested methanol showed the most promise. 


\subsection{Neutral Salt in a Non-aqueous Medium}

A stronger apparent proton activity should be observed if the dehydration effect achieved by the addition of the neutral cation is combined with titration in a non-aqueous medium. It was decided to combine the two approaches: that of the addition of a neutral salt, and the use of a non-aqueous solvent.

From the non-aqueous titrations methanol showed the most promise and was also the cheapest solvent tested. It was therefore chosen as the solvent for this test. Magnesium chloride exhibited the desired neutral salt effect, it was therefore dissolved in the methanol. The sample was added to $50 \mathrm{ml}$ of methanol in which magnesium chloride was dissolved.

Different concentrations of magnesium chloride in methanol were tested and it was found that the inflection of the titration curve improved markedly with the $80 \%$ magnesium chloride in methanol solution (Figure 4.6). Sodium hydroxide was dissolved in methanol and used as the titrant to see if this offered any improvement to the titration curve. The sodium hydroxide formed a cloudy precipitate in methanol. The undissolved particles were allowed to settle and the supernatant decanted. During the titration there was a large number of bubbles present and mixing was not adequate. The inflection for this curve was not as clearly defined as the sodium hydroxide in water curve as seen in Figure 4.7. Sodium hydroxide in methanol offers no benefit over sodium hydroxide in water to the resolution of the endpoints. 
Figure 4.6: Acid titration curves in presence of variable magnesium chloride concentrations.
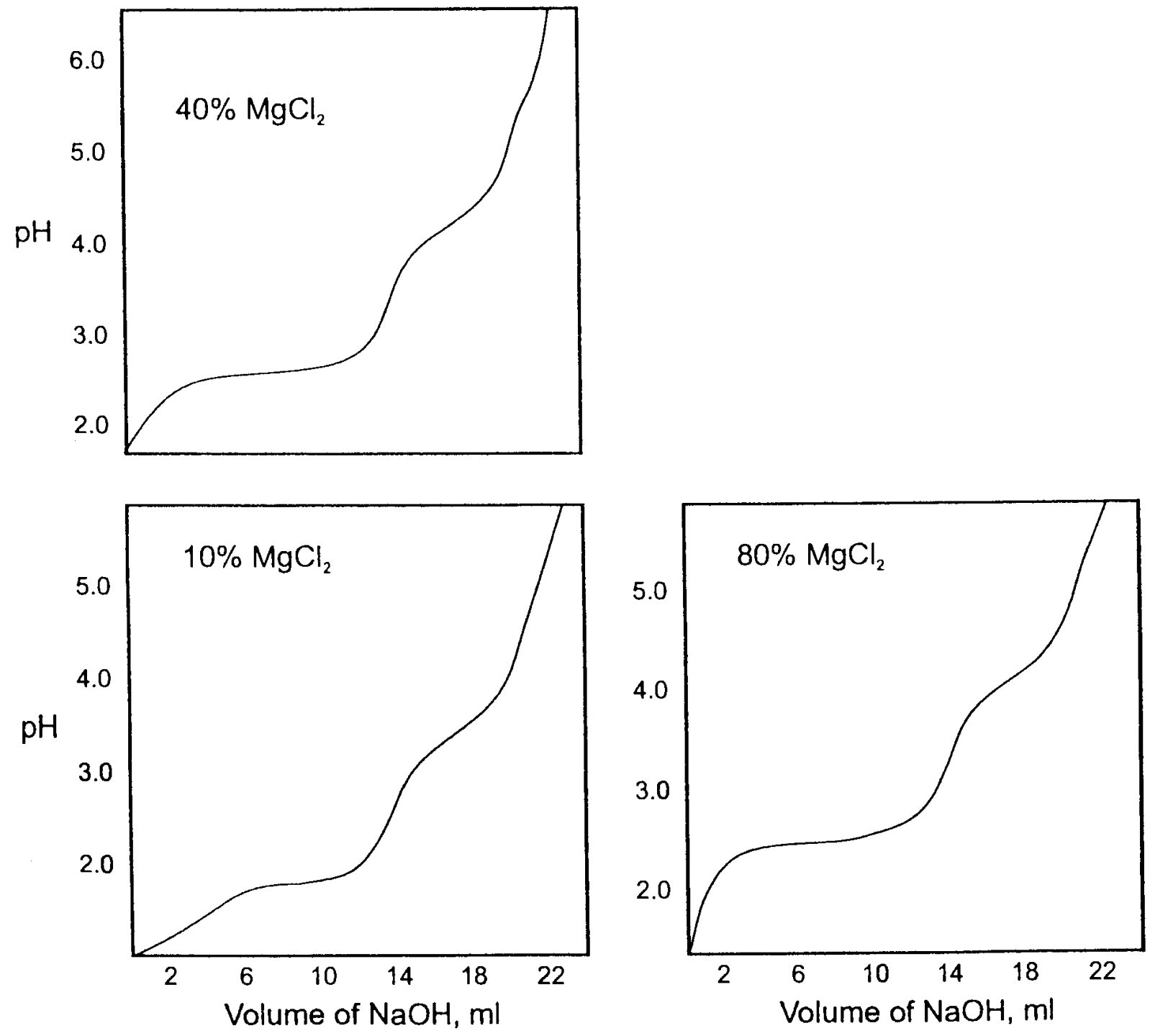
Figure 4.7: Comparison of titrations using sodium hydroxide in methanol and sodium hydroxide in water.

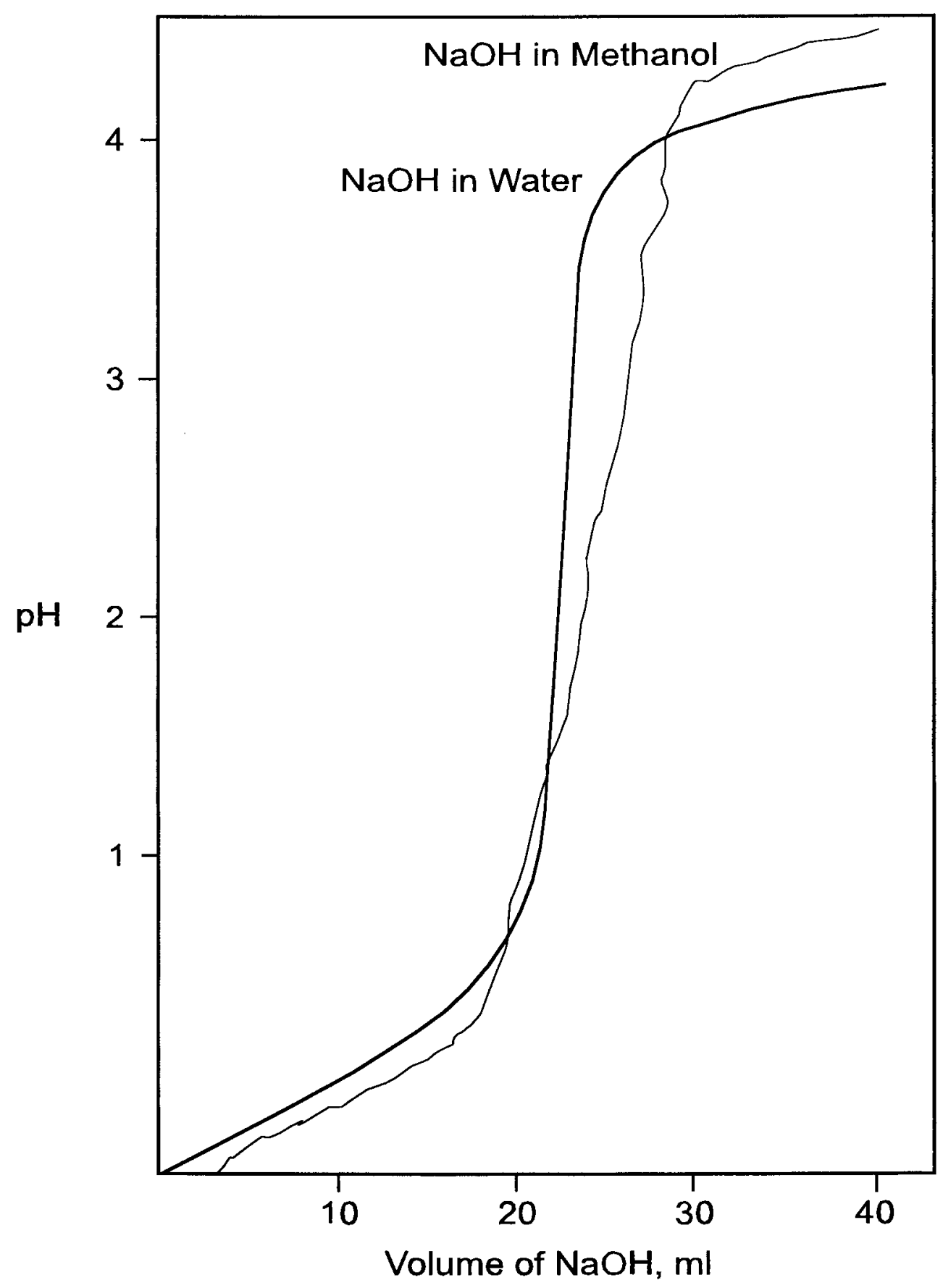




\subsubsection{Conclusion}

The combination of the two approaches: that of the addition of a neutral salt, and the use of a non-aqueous solvent, produces a stronger apparent proton activity. A non-aqueous solution of $80 \%$ magnesium chloride in methanol was found to be the best solution to use in the determination of acid in the presence of hydrolysable cations. 


\subsection{Analysis of Samples, Detection Limits}

It was found that for the most serious interferents $4.8 \mathrm{~g} / \mathrm{l}$ of sulphuric acid could be determined in the presence of $90.3 \mathrm{~g} / 1$ of iron(III) and $200 \mathrm{~g} / \mathrm{l}$ of manganese(II). It became apparent during these determinations that the aliquot size taken for a determination influence the detection limit to a great extent. A zinc leaching sample (HD 987/1) containing $22.0 \mathrm{~g} / 1$ of iron and zinc feed samples (GF 802 and 809 ) containing approximately $12 \mathrm{~g} / 1$ of iron and $10 \mathrm{~g} / 1$ of copper, were titrated using magnesium chloride as the neutral salt and the results were compared to those obtained using lithium chloride and are displayed in Table 4.19. From these analyses it became evident that the size of sample aliquot is important in obtaining good accuracy and precision.

Table 4.19: Influence of magnesium chloride on the free acid results

\begin{tabular}{|l|l|l|l||}
\hline Sample & $\begin{array}{l}{\left[\mathrm{H}_{2} \mathrm{SO}_{4}\right](\mathrm{g} / \mathrm{l}) \text { with }} \\
\mathbf{M g C l}_{2}\end{array}$ & $\begin{array}{l}\text { \% Spike Recovery } \\
\text { using } \mathbf{M g C l}_{2}\end{array}$ & $\begin{array}{l}{\left[\mathrm{H}_{2} \mathrm{SO}_{4}\right](\mathrm{g} / \mathbf{l}) \text { with }} \\
\mathbf{L i C l}\end{array}$ \\
\hline GF 802/1 & 24.63 & 101 & 24.71 \\
\hline GF 802/2 & 54.53 & 90 & 54.61 \\
\hline GF 809/1 & 3.64 & 93 & 4.00 \\
\hline GF 809/5 & 21.85 & 91 & 23.88 \\
\hline GF 809/6 & 21.18 & 92 & 19.76 \\
\hline HD 987/1 & 39.26 & 95 & 39.63 \\
\hline
\end{tabular}


It became apparent during these determinations that the aliquot size taken for a determination influence the detection limit to a great extent. It is necessary that when a sample with unknown acid and cation concentration has to be analysed, tests are done to approximate the acid content and to see whether there is enough acid present to be detected. If the acid content is not sufficient, larger aliquots should be taken and tested to see if the acid can be detected. Larger aliquots don't always imply that the acid level could be detected, since the level of interfering cations is also increased. Each sample has to be analysed on merit and a careful examination of the resulting neutralisation curve made in order to determine if the analysis is possible or not.

If there is doubt regarding the success of the analysis then a sample spike recovery should be carried out [24]. A spike recovery is the addition of a known amount of the analyte to be determined. The difference of the result of the sample and the added analyte, compared to the sample alone gives the recovery of the analyte added. This indicates whether the method is carried out correctly as well as if there is any interference present. For quality purposes a sample spike recovery should be carried out on different samples analysed to ascertain suitability of the method to the sample type.

\subsubsection{Conclusion}

For $4 \mathrm{~g} / 1$ of acid solution up to $54 \mathrm{~g} / 1$ iron(III) can be tolerated. The tolerance levels depend to a large extent on the aliquot taken as well as the presence of other interfering cations in the sample solutions. For solutions with unknown cation concentrations the 
samples need to be tested and the neutralisation curves evaluated for interferences at the acid endpoint. If there is still doubt the sample solutions to be analysed should be spiked to determine any possible interference. 


\subsection{Figures of Merit}

The magnesium chloride in methanol method of dealing with the interferences was chosen as the most appropriate method. The method was then statistically evaluated to determine the figures of merit.

\subsubsection{Accuracy}

The accuracy of a determination is defined as the agreement between the determined value and the true or most probable value [24,31]. For this study the absolute method was used to determine the accuracy of the procedure under question.

Synthetic samples containing known amounts of the interferences in question with known sulphuric acid concentration were prepared. The difference between the mean of a number of results and the amount of the sulphuric acid actually present is a measure of the accuracy of the method. 
Table 4.20: Relative Error of various solutions using titrations

\begin{tabular}{|l|l|l||}
\hline [Cation] in g/l & Mean Result & \% Relative Mean Error \\
\hline $127 \mathrm{Co}^{2+}$ & $4.72(\mathrm{n}=10)$ & -2.54 \\
\hline $204 \mathrm{Mn}^{2+}$ & $4.60(\mathrm{n}=10)$ & 13.77 \\
\hline $171 \mathrm{Ni}^{2+}$ & $5.37(\mathrm{n}=10)$ & -1.91 \\
\hline $38 \mathrm{Fe}^{2+}$ & $4.63(\mathrm{n}=10)$ & - \\
\hline $53 \mathrm{Cu}^{2+}$ & No endpoint $(\mathrm{n}=10)$ & 0.85 \\
\hline $90 \mathrm{Fe}^{3+}$ & $4.76(\mathrm{n}=10)$ & - \\
\hline
\end{tabular}

Table 4.21: Relative Error of various solutions using the magnesium chloride method

\begin{tabular}{|l|l|l||}
\hline [Cation] in g/l & Mean Result & \% Relative Mean Error \\
\hline & $4.86(\mathrm{n}=10)$ & \\
\hline $127 \mathrm{Co}^{2+}$ & $5.14(\mathrm{n}=10)$ & 5.76 \\
\hline $204 \mathrm{Mn}^{2+}$ & $5.43(\mathrm{n}=10)$ & 11.73 \\
\hline $171 \mathrm{Ni}^{2+}$ & $4.88(\mathrm{n}=10)$ & 0.42 \\
\hline $38 \mathrm{Fe}^{2+}$ & $5.21(\mathrm{n}=10)$ & 7.20 \\
\hline $53 \mathrm{Cu}^{2+}$ & $4.76(\mathrm{n}=10)$ & -2.06 \\
\hline $90 \mathrm{Fe}^{3+}$ & $4.93(\mathrm{n}=10)$ & 1.44 \\
\hline
\end{tabular}


For the purposes of providing accurate results, within MINTEK's quality system which is based on the SABS 0259 laboratory accreditation system, it was agreed that a relative error of up to $10 \%$ is acceptable. A comparison of Table 4.20 and 4.21 would indicate that there is a definite improvement in accuracy of the method when using magnesium chloride in methanol. There is a slight decrease in accuracy for nickel(II) and copper(II). This decrease in accuracy becomes negligible when compared to the increase in accuracy for the other cations. It would seem that the accuracy of the determinations in the presence of manganese falls just short of the $10 \%$ acceptable relative error. It should be born in mind that these determinations were done on manganese nitrate which gave a large interference when compared to the manganese chloride. There is a $2.1 \%$ improvement in the relative error when comparing the two methods. It should be noted that acid determinations in the presence of manganese nitrate would yield results that would fall outside the $10 \%$ acceptable relative error.

\subsubsection{Precision}

Precision is defined as the agreement between two or more measurements that have been made and is a measure of reproducibility of a method [24,31]. Precision accompanies accuracy, but a high degree of precision does not imply accuracy. Relative standard deviation is a measure of precision (equation 4.10).

$$
\text { Relative Standard Deviation R.S.D. }=\frac{\left\{\left[\sum\left(\mathrm{x}_{\underline{I}}-\overline{\mathrm{x}}\right)^{2}\right] /(\mathrm{n}-1)\right\}^{1 / 2}}{\mathrm{n}-1}
$$


Table 4.22: R.S.D. values for the various interferents

\begin{tabular}{||l|l||}
\hline [Cation] in g/l & R.S.D. \\
\hline $127 \mathrm{Co}^{2+}$ & 1.33 \\
\hline $204 \mathrm{Mn}^{2+}$ & 1.35 \\
\hline $171 \mathrm{Ni}^{2+}$ & 1.75 \\
\hline $90 \mathrm{Fe}^{3+}$ & 3.40 \\
\hline $53 \mathrm{Cu}^{2+}$ & 1.66 \\
\hline $34 \mathrm{Fe}^{2+}$ & 5.43 \\
\hline
\end{tabular}

The R.S.D. values indicate a great degree of precision for the determinations in the presence of the individual cations. Even though the determinations in the presence of manganese showed a large percentage of inaccuracy they show a good precision. It would appear that there is a source of constant error present in the acid determinations in the presence of manganese. It is however not possible to quantify this error since it would have to be done for every concentration of manganese possible as well as for every acid concentration possible and then both concentrations would have to be known before results can be corrected for the error. This is not a convenient solution. 


\subsubsection{Conclusion}

An improvement in accuracy is noted from the titration to the titration using magnesium chloride in methanol. All the titrations gave accuracies within the required limit except the manganese(II) titrations that indicate an accuracy just outside the accepted limit. The overall improvement in accuracy still makes the magnesium chloride in methanol a useful method. All the titrations using the magnesium chloride in methanol are very precise. 


\section{Chapter 5: Conclusion}

This project was initiated to overcome interferences from hydrolysable cations in the acid determinations in samples that originated from hydrometallurgical processes with high cation concentrations. The samples analysed contained iron(II), iron(III), cobalt(II), copper(II), nickel(II), and manganese(II). During the acid-base titration, these hydrolysable cations form basic salts or hydroxides, releasing protons, which consume part of the titrant. With conventional methods the loss of titrant due to this hydrolysation is indistinguishable from the titrant necessary for the neutralisation of the sulphuric acid, and the titrations gave erroneously high acid values. The problem is magnified as the ratio of hydrolysable cation to free acid increases above one.

Various methods have been reported to determine the acid concentration in the presence of hydrolysable cations. The methods are however usually specific to certain defined matrices. Furthermore the methods are only able to tolerate a iron(III) to free acid ratio of 2:1. From the reported methods the ones chosen for exploration in this study can be broadly classified into the following:

1. Addition of a complexometric reagent- to mask the hydrolysis of the cation.

2. Addition of neutral salts- to enhance the activity of hydrogen ions.

Solutions containing the cations present in the hydrometallurgical samples were spiked with sulphuric acid and the free acid concentration was determined. The most serious interference was encountered with iron(III) and manganese(II). Manganese nitrate causes 
a more significant interference than manganese chloride because nitrate undergoes spontaneous reduction in the presence of manganese(II). Copper(II), cobalt(II), and nickel(II) present no real problems. Although for highly coloured solutions a potentiometric titration is preferred to a colourimetric titration. Solutions containing iron(II) should be treated with care to ensure that no oxidation occurs prior to titration.

Using potassium tartrate and potassium oxalate to complex iron(III) gave inaccurate results even though thermodynamic considerations indicate that complexometric reagents should mask the effects of iron(III). This apparent discrepancy between theory and practise may be due to differences in concentrations and ionic strengths existing between 'real' solutions.

Lithium chloride was suggested as neutral salt for the determination of free acid concentration in the presence of hydrolysable cations. It was suggested that the presence of a neutral salt enhances the apparent $\mathrm{pH}$ of a solution and improves the shape of the inflections in the potentiometric curves. The conditions for the use of lithium chloride was optimised. With the use of lithium chloride the free acid determinations on the presence of the various cations gave satisfactory results. However, the cost of lithium chloride inhibits its routine use and a cost-effective alternative had to be found.

Tests indicated that both the lithium ion and the chloride ion aid in shifting the hydrolysis of the hydrolysable cations present to a higher $\mathrm{pH}$. If an alternative neutral salt was to be selected it would have had to have both a suitable cation and a chloride ion. 
In order to find a neutral salt that would behave similar to lithium chloride factors such as heat of solution, solubility, and charge to size ratio were taken considered. Magnesium chloride was chosen for further investigation. It gave results comparable to that obtained with lithium chloride and hence was chosen as an alternative to lithium chloride.

Since the problems experienced during the free acid titrations are influenced by cation hydration it was thought that anhydrous solvents would theoretically have the same effect as high concentrations of neutral salts by inhibiting the hydration of the hydrolysable cations. In some instances the non-aqueous titrations showed some promise, although the curves were not always as clearly defined as that obtained in aqueous titrations. Methanol showed the most promise of the organic solvents tested.

It was decided to combine the two approaches: addition of a neutral salt, and the use of a non-aqueous solvent. A stronger apparent proton activity was produced which resulted in a greater resolution of the proton and cation endpoints. Hence there was a better tolerance of cations.

The combination of neutral salt in non-aqueous medium worked well to overcome the interference of hydrolysable cations. $80 \%$ magnesium chloride in methanol worked particularly well. A high tolerance of hydrolysable cations (up to a 20 fold excess) without interference during the titration of very low concentrations of sulphuric acid, with good accuracy and precision was found. In addition for routine use, magnesium 
chloride is preferred to lithium chloride as it is more cost-effective (magnesium chloride costs 30 times less than the equivalent amount of lithium chloride). 


\section{References}

1. Dry MJ, Iorio G, Jacobs DF, Cole PM, Feather AM, Sole KC, Engelbrecht J, Matchett KC, Cilliers PJ, O'Kane PT, Dreisinger DB (1998), Cu/ Co tailings treatment project, Democratic Republic of Congo. ALTA Ni/ Co pressure leaching \& hydrometallurgy forum. Perth, Australia

2. Britton HTS, (1955) Hydrogen Ions (Volume 1), Chapman and Hall, Great Britain

3. Britton HTS, (1955) Hydrogen Ions (Volume 2), Chapman and Hall, Great Britain

4. Solomons M (1980) Mintek Report 2039. Mintek, Randburg

5. Mayankutty PC, Ravi S, Nadkarni MN (1982) J Radioanal Chem 68:145

6. Baumann EW, Torrey BH (1984) Anal Chem 56:682

7. Barsewisch EHR, Hasty RA (1985) Mintek Report M194. Mintek, Randburg

8. Bulatov MI, Bulatov AA (1983) Zh Anal Khim 38:1607

9. Prassad TP (1991) Res Ind 36:22

10. Rossotti FJC, Rossotti H (1965) J Chem Ed 42:375

11. Pakalns P (1981) Analytica Chimica Acta, 127:263

12.Booman GL, Elliott MC, Kimball RB, Cartan FO, Rein JE, (1958) Analytical Chemistry, 30:284

13. Critchfield FE, Johnson JB (1959) Anal Chem 31:570

14. Critchfield FE, Johnson JB (1958) Anal Chem 30:1247

15. Kubota A, Costanzo DA (1964) Anal Chem $36: 2454$

16. Hogfeldt E, Leifer L (1963) Acta Chem Scan 17:338

17. Gran G, (1950) Acta Chem Scand 4:559 
18. Gran G, (1952) Part II, Analyst, 77:661

19. Stumm W, Morgan JJ, (1970) Aquatic Chemistry, John Wiley and Sons, USA

20.Editiors Kroschwitz JI, Howe-Grant M, (1997) Kirk-Othmer Encyclopaedia of Chemical Technology ( $4^{\text {th }}$ Edition) John Wiley and Sons, USA

21.Editors Bartlett N, et al, (1992) Encyclopaedia of Science and Technology $\left(7^{\text {th }}\right.$ Edition) McGraw Hill, USA

22. Editors Weast RC, Astle MJ, (1982) CRC Handbook of Chemistry and Physics (Sixty Second Edition), CRC Press, USA

23. Sander UHF, Fischer H, Rothe U, Kola R, (1984) Sulphur, Sulphur Dioxide and Sulphuric Acid, Verlag Chemie, London

24.Basset J, Denny RC, Jeffery GH, Mendham J (1979) Vogel's Textbook of Quantitative Inorganic Chemistry (Fourth edition). Pergamon Press, London

25. Masterton WL, Slowinski EJ (1977) Chemical principles (Fourth edition) Saunders Press, USA

26. Monroe M, Abrams K (1985) J Chem Ed 62:41

27. Huheey JE (1978) Inorganic Chemistry (Second edition). Harper International, USA

28. Mattock G, Taylor GR (1961) pH Measurement and Titration. Haywood and Co, London

29. Blaedel WJ, Meloche VW (1964) Elementary Qualitative Analysis (Second edition), Harper International, USA

30. Peters DG, Hayes JM, Hieftje GM, (1976) A Brief Introduction to Modern Chemical Analysis, Saunders, USA 
31.Skoog DA, West DM, Holler FJ, (1992) Fundamentals of Analytical Chemistry (Sixth Edition), Saunders, USA

32. Kolthoff IM, Stenger VA, (1947) Volumetric Analysis (Volume 1,2), Interscience, New York

33. Editors Kolthoff IM, Elving PJ, Sandell EB, (1978) Treatise on Analytical Chemistry, Interscience, USA

34. Cotton FA, Wilkinson G, Gaus PL, (1987) Basic Inorganic Chemistry ( ${ }^{\text {rd }}$ edition), John Wiley and Sons,USA

35. Kolthoff IM, Furman NH, (1947) Potentiometric Titrations, John Wiley and Sons, USA

36. Metrosensor Electrodes, Metrohm, Switzerland

37. Valcarcel M, de Castro MD, (1988) Automatic Methods of Analysis, Elsevier, USA

38. Martell AE, Smith RM, (1977) Other Organic Ligands (Volume3), Plenum Press, USA

39. Ringbom A (1963) Complexation in Analytical Chemistry, Interscience, London

40. Moeller T (1957) Inorganic Chemistry, John Wiley and Sons, USA

41. Metrohm, Potentiometric analysis of nitric acid. Application Bulletin no. 039/3e. Metrohm, Switzerland

42.Fritz JS (1973) Acid-base Titrations in Non-aqueous Solvents, Allyn and Bacon, USA

43. Zelenka L, Sak-Bosnar M, Pavosevic Z (1988) J Serb Chem Soc 53:219

44. Poltevskii GV, Yakushin AI, Savelyanov VP (1977) Zh Anal Khim 32:638

45. Merck Tables for the Laboratory, Merck, Germany 